\title{
Direct Imaging discovery of a second planet candidate around the possibly transiting planet host CVSO $30^{\star}$
}

\author{
T. O. B. Schmidt ${ }^{1,2}$, R. Neuhäuser ${ }^{2}$, C. Briceño ${ }^{3}$, N. Vogt $^{4}$, St. Raetz $^{5}$, A. Seifahrt ${ }^{6}$, C. Ginski ${ }^{7}$, M. Mugrauer $^{2}$, \\ S. Buder ${ }^{2,8}$, C. Adam ${ }^{2}$, P. Hauschildt ${ }^{1}$, S. Witte ${ }^{1}$, Ch. Helling 9 , and J. H. M. M. Schmitt ${ }^{1}$ \\ ${ }^{1}$ Hamburger Sternwarte, Gojenbergsweg 112, 21029 Hamburg, Germany \\ e-mail: tschmidt@hs.uni-hamburg.de \\ 2 Astrophysikalisches Institut und Universitäts-Sternwarte, Universität Jena, Schillergäßchen 2-3, 07745 Jena, Germany \\ 3 Cerro Tololo Inter-American Observatory CTIO/AURA/NOAO, Colina El Pino s/n. Casilla 603, 1700000 La Serena, Chile \\ ${ }^{4}$ Instituto de Física y Astronomía, Universidad de Valparaíso, Avenida Gran Bretaña 1111, 2340000 Valparaíso, Chile \\ 5 European Space Agency ESA, ESTEC, SRE-S, Keplerlaan 1, 2201 AZ Noordwijk, The Netherlands \\ 6 Department of Astronomy and Astrophysics, University of Chicago, 5640 S. Ellis Ave., Chicago, IL 60637, USA \\ 7 Sterrewacht Leiden, PO Box 9513, Niels Bohrweg 2, 2300 RA Leiden, The Netherlands \\ 8 Max-Planck-Institute for Astronomy, Königstuhl 17, 69117 Heidelberg, Germany \\ 9 School of Physics and Astronomy SUPA, University of St. Andrews, North Haugh, St. Andrews, KY16 9SS, UK
}

Received 16 April 2015 / Accepted 14 March 2016

\begin{abstract}
Context. Direct imaging has developed into a very successful technique for the detection of exoplanets in wide orbits, especially around young stars. Directly imaged planets can be both followed astrometrically on their orbits and observed spectroscopically and thus provide an essential tool for our understanding of the early solar system.

Aims. We surveyed the 25 Ori association for direct-imaging companions. This association has an age of only few million years. Among other targets, we observed CVSO 30, which has recently been identified as the first T Tauri star found to host a transiting planet candidate.

Methods. We report on photometric and spectroscopic high-contrast observations with the Very Large Telescope, the Keck telescopes, and the Calar Alto observatory. They reveal a directly imaged planet candidate close to the young M3 star CVSO 30.

Results. The $J H K$-band photometry of the newly identified candidate is at better than $1 \sigma$ consistent with late-type giants, early-T and early-M dwarfs, and free-floating planets. Other hypotheses such as galaxies can be excluded at more than 3.5 $\sigma$. A lucky imaging $z^{\prime}$ photometric detection limit $z^{\prime}=20.5$ mag excludes early-M dwarfs and results in less than $10 M_{\text {Jup }}$ for CVSO $30 \mathrm{c}$ if bound. We present spectroscopic observations of the wide companion that imply that the only remaining explanation for the object is that it is the first very young $(<10 \mathrm{Myr}) \mathrm{L}-\mathrm{T}$-type planet bound to a star, meaning that it appears bluer than expected as a result of a decreasing cloud opacity at low effective temperatures. Only a planetary spectral model is consistent with the spectroscopy, and we deduce a best-fit mass of 4-5 Jupiter masses (total range 0.6-10.2 Jupiter masses).

Conclusions. This means that CVSO 30 is the first system in which both a close-in and a wide planet candidate are found to have a common host star. The orbits of the two possible planets could not be more different: they have orbital periods of $10.76 \mathrm{~h}$ and about $27000 \mathrm{yr}$. The two orbits may have formed during a mutual catastrophic event of planet-planet scattering.
\end{abstract}

Key words. stars: pre-main sequence - stars: low-mass - planetary systems - planets and satellites: detection planets and satellites: atmospheres - planets and satellites: formation

\section{Introduction}

Since the first definite detection of a planet around another main-sequence star, 51 Peg (Mayor \& Queloz 1995) made with high-precision radial velocity measurements, various detection techniques have been applied to find a diverse population of exoplanets. The transit method, which was first used for HD 209458 (Charbonneau et al. 2000), later allowed for a boost of exoplanet discoveries after the successful launch of two dedicated satellite missions, CoRoT (Baglin et al. 2007) and Kepler (Koch et al. 2010; Borucki et al. 2010). These two methods indirectly discern the presence of a planet by the influence

\footnotetext{
* Based on observations made with ESO Telescopes at the La Silla Paranal Observatory under programme IDs 090.C-0448(A), 290.C-5018(B), 092.C-0488(A) and at the Centro Astronómico Hispano-Alemán in programme H15-2.2-002.
}

the planet has on its host star. The methods are most sensitive to small and moderate planet-star-separations around old mainsequence stars that are fairly inactive because of their age. The sensitivity diminishes fast for separations beyond 5 au because transits become less likely and the radial velocity amplitude declines with increasing orbital period. In contrast, direct imaging allows discovering planets on wide orbits around nearby premain-sequence stars because such young planets are still bright at infrared wavelengths as a result of the gravitational contraction during their still-ongoing formation process.

The total number of imaged planet candidates has increased to about 50-60 objects today. The first detections were made in 2005 , when the first four co-moving planetary candidates were found around the solar-like stars DH Tau (Itoh et al. 2005), GQ Lup (Neuhäuser et al. 2005), and AB Pic (Chauvin et al. 2005c), all with masses near the threshold of $13 M_{\text {Jup }}$ that divides brown 


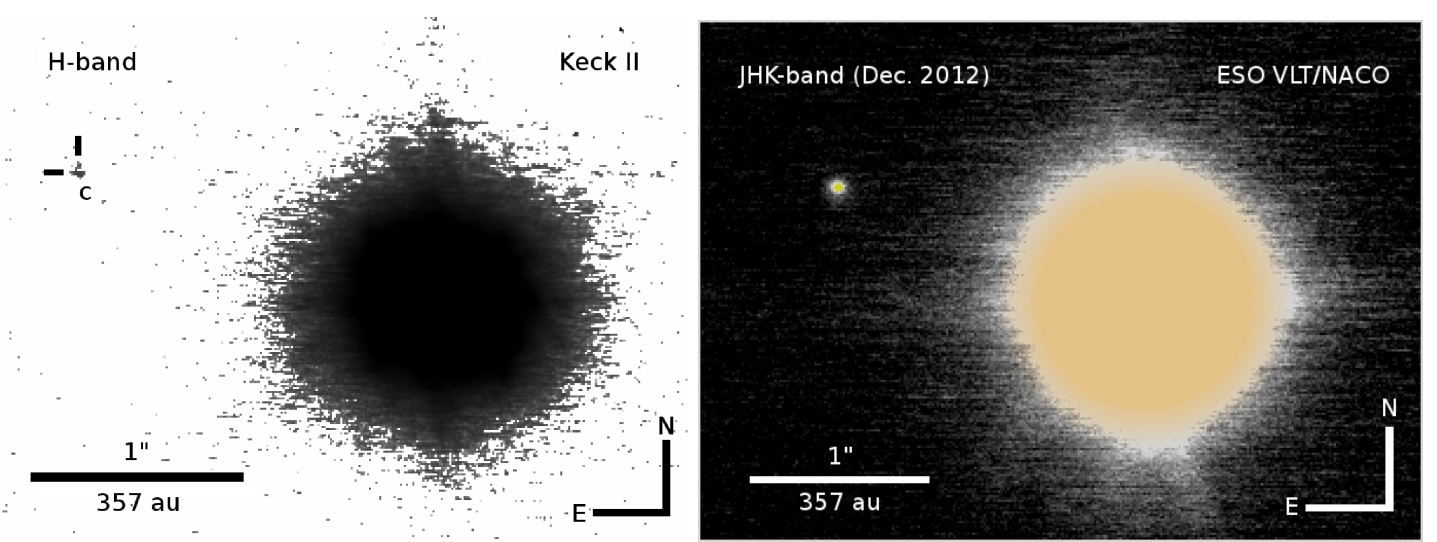

Fig. 1. Direct images of CVSO 30 c. Left: Keck image of data by van Eyken et al. (2012), re-reduced. We note that the companion is north-east and not a contaminant south-east, as given in van Eyken et al. (2012). Right: our new VLT epoch, clearly showing the planetary companion, which has similar colour as its host star (Fig. 2). This excludes that it is a false positive for the inner planet candidate CVSO $30 \mathrm{~b}$.

dwarfs from planets according to the current IAU working definition, together with the planet candidate around the brown dwarf 2M1207 (Chauvin et al. 2005a). A summary can be found in Neuhäuser \& Schmidt (2012), and the current status is always available in several online encyclopaediae, such as the Extrasolar Planets Encyclopaedia at www . exoplanet . eu (Schneider et al. 2011). As in situ formation at $\sim 100$ au to a few hundreds of au separation seems unlikely according to models, Boss (2006) argued that a third body must exist that tossed these planets outward to their present distance from their young host stars. An alternative explanation might be a stellar encounter (Adams \& Laughlin 2001).

While early-type stars have less favourable planet-to-star contrast ratios, increasing evidence was found by millimetercontinuum measurements for larger and more massive protoplanetary disks that are available for planet formation around these stars (Mannings \& Sargent 1997; Andrews et al. 2013). These conclusions were further strengthened when three of the most prominent planet candidates were found in 2008 and 2009 around the early F-type star HR 8799 (Marois et al. 2008), which is the first system with multiple planets imaged around a star. The other two were around the A-type stars Fomalhaut (Kalas et al. 2008), which is the first planet candidate discovered in the optical regime using the Hubble Space Telescope (HST), and $\beta$ Pic (Lagrange et al. 2009, 2010), a planet within the large edge-on disk at only about twice the separation of Jupiter from the Sun. This had previously been predicted by Freistetter et al. (2007), for instance, from the structural gaps in the disk.

Most of the direct-imaging surveys conducted so far have concentrated on AFGK stars. In 2012 a (proto-)planet candidate was discovered around the $\sim 2 \mathrm{Myr}$ young solar-like star $\mathrm{LkCa}$ 15 (Kraus \& Ireland 2012). This is a close ( $\sim 11$ AU) object found by single-dish interferometry, which is a technique that is also referred to as sparse aperture masking. Two companions of 4-5 $M_{\text {Jup }}$ were recently discovered around GJ 504 (Kuzuhara et al. 2013), which is a $160 \mathrm{Myr}$ old solar-like star, and around HD 95086 (Rameau et al. 2013), an A-type star at about 10 17 Myr. Additionally, first results from imaging surveys around $\mathrm{M}$ dwarfs were published during the past two years, which increased our understanding of planetary systems around the most numerous stars in the Milky Way (Delorme et al. 2013; Bowler et al. 2015).

In this article we describe for the first time the direct detection of a wide-separation (1.85" or $662 \mathrm{au}$, see Fig. 1) directly imaged planet candidate around a star (CVSO 30) that also hosts a short-period transiting planet candidate; we refer to a more detailed discussion of this object in van Eyken et al. (2012), Barnes et al. (2013), Yu et al. (2015), and Raetz et al. (2016). A system that harbours two planets with such extreme orbits gives us for the first time the opportunity to study by observations the possible outcome of planet-planet scattering theories, which have been used to explain the existence of close-in hot Jupiters in 1996 (Rasio \& Ford 1996).

\section{25 Ori group and the CVSO 30 system properties}

Despite their importance for the evolution of protoplanetary disks and the early phases in the planet formation process, sufficiently large samples of $10 \mathrm{Myr}$ old stars have been difficult to identify, mainly because the parent molecular clouds dissipate after a few Myr and no longer serve as markers of these populations (see Briceño et al. 2007b and references therein). The 25 Ori cluster (25 Ori, Briceño et al. 2007a) contains >200 PMS stars in the mass range $0.1<M / M_{\odot}<3$. The Hipparcos OB and earlier A-type stars in 25 Ori are on the zero-age main sequence (ZAMS, Hernández et al. 2005), implying a distance of $\sim 330 \mathrm{pc}$, with some of the A-type stars harbouring debris disks (Hernández et al. 2006). Isochrone fitting of the low-mass stars yields an age of 7-10 Myr (Briceño et al. 2007b). This is the most populous $10 \mathrm{Myr}$ old sample within $500 \mathrm{pc}$, which we consequently chose for a direct-imaging survey with ESO's VLT, the Very Large Telescope of the European Southern Observatory, to find young planetary and sub-stellar companions at or shortly after their formation. For this same reason, the 25 Ori cluster was also targeted in searches for transiting planets, like the Young Exoplanet Transit Initiative (YETI, Neuhäuser et al. 2011) and the Palomar Transient Factory (PTF, van Eyken et al. 2012).

CVSO 30 (also 2MASS J05250755+0134243 and PTFO 8-8695) is a weak-line T Tauri star of spectral type M3 in 25 Ori at an average distance of $357 \pm 52$ pc (Downes et al. 2014). It was confirmed as a T Tauri member of the 25 Ori cluster by the CIDA Variability Survey of Orion (CVSO), with properties shown in Table 1. As shown in Fig. 1 in van Eyken et al. (2012), CVSO 30 is one of the youngest objects within 25 Ori, its position in the colour-magnitude diagram corresponds to $2.39_{-2.05}^{+3.41} \mathrm{Myr}$ (if compared to evolutionary models of Siess et al. 2000). The object is highly variable, rotates fast, and has a mass of $0.34-0.44 M_{\odot}$ (depending on the evolutionary model), and an effective temperature of $\sim 3470 \mathrm{~K}$. The rotation period of CVSO 30, which is possibly synchronised with the CVSO $30 \mathrm{~b}$ 
T. O. B. Schmidt et al.: Direct Imaging of a second planet candidate in the transiting CVSO 30 system

orbital period, is still debated (van Eyken et al. 2012; Koen 2015). Kamiaka et al. (2015) concluded that the stellar spin period is shorter than $0.671 \mathrm{~d}$.

In 2012 the PTF team (van Eyken et al. 2012) reported a young transiting planet candidate around CVSO 30, named PTFO 8-8695 b, with a fast co-rotating or near corotating 0.448413 day orbit. The very same object, henceforth CVSO $30 \mathrm{~b}$ for simplicity, was independently detected with smaller telescopes within the YETI (Neuhäuser et al. 2013; Errmann et al. 2014), confirming the presence of the transit events by quasi-simultaneous observations.

Keck and Hobby-Eberly Telescope (HET) spectra (van Eyken et al. 2012) set an upper limit to the mass of the transiting companion of $5.5 \pm 1.4 M_{\text {Jup }}$ from the radial velocity variation, which exhibits a phase offset that is likely caused by spots on the surface of the star. This RV limit was already corrected for the derived orbital inclination $61.8 \pm 3.7^{\circ}$ of the system. With an orbital radius of only about twice the stellar radius and a planetary radius of $1.91 \pm 0.21 R_{\text {Jup }}$, the object appears to be at or within its Roche-limiting orbit, raising the possibility of past or ongoing mass loss. A false positive by a blended eclipsing binary is unlikely because the only present contaminant in Keck near-IR images (see Fig. 1) with 6.96 mag of contrast to the star would have to be very blue to be bright enough in the optical to mimic a transit; it is unlikely to be a star in that case.

In 2013 Barnes et al. (2013) fit the two separate light curves observed in 2009 and 2010, which exhibited unusual differing shapes, simultaneously and self-consistently with planetary masses of the companion of 3.0-3.6 $M_{\text {Jup }}$. They assumed transits across an oblate, gravity-darkened stellar disk and precession of the planetary orbit ascending node. The fits show a high degree of spin-orbit misalignment of about $70^{\circ}$, which leads to the prediction that transits disappear for months at a time during the precession period of this system. The lower planet radius result of $\sim 1.65 R_{\text {Jup }}$ is consistent with a young, hydrogen-dominated planet that results from hot-start formation mechanisms (Barnes et al. 2013).

\section{Astrometric and photometric analysis}

After the discovery of the transiting planet candidate by van Eyken et al. (2012) and our independent detection of the transit signals with YETI, we included the system in our 25 Ori VLT/NACO direct-imaging survey with the intent to prove that the object labelled as a contaminant by van Eyken et al. (2012) is not able to produce the detected transiting signal and to confirm it as second planet. We performed our first highresolution direct observations in December 2012 and obtained $J H K$-band photometry (Tables 2 and 3 and Fig. 1).

During the course of their study of the transiting planet CVSO $30 \mathrm{~b}$, the PTF team used Keck II/NIRC2 $H$-band images obtained in 2010 to identify contaminants capable of creating a false-positive signal mimicking a planet. We re-reduced these data and found that they already contain the planetary companion CVSO $30 \mathrm{c}$ that we report here. In Fig. 1 we show the companion, erroneously given to lie south-east in van Eyken et al. (2012); it is located north-east of the host star CVSO 30.

We astrometrically calibrated the VLT/NACO detector epoch using a sub-field of 47 Tuc (Table 5) to determine pixel scale and detector orientation in order to find precise values for the separation of CVSO $30 \mathrm{c}$ with respect to CVSO 30 in right ascension and declination. From this, we find the object to be $\sim 1.85^{\prime \prime} \mathrm{NE}$ of CVSO 30 at a position angle of $\sim 70^{\circ}$ from north towards east, corresponding to a projected separation of $662 \pm 96$ au at the
Table 1. Previously known CVSO 30 system data.

\begin{tabular}{|c|c|}
\hline & CVSO 30 \\
\hline Altern. designations & 2MASS J05250755+0134243, PTF1 J052507.55+013424.3 \\
\hline Location & 25 Ori/Orion OB 1a $[1,2]$ \\
\hline RA, Dec & $05 \mathrm{~h} 25 \mathrm{~m} 07.57 \mathrm{~s},+01^{\circ} 34^{\prime} 24.5^{\prime \prime}[2]$ \\
\hline Spectral type & M3 (weak-line T Tauri, WTTS) [2] \\
\hline Mass & $0.34 / 0.44 M_{\odot}[2]$ \\
\hline Luminosity & $0.25 L_{\odot}[2]$ \\
\hline Radius & $1.39 R_{\odot} / 1.07 \pm 0.10 R_{\odot} /\left[1.03 / 1.04 \pm 0.01 R_{\odot}\right][2,3,4]$ \\
\hline Temperature & $3470 \mathrm{~K}[2]$ \\
\hline Opt. extinction & $0.12 \mathrm{mag}$ [2] \\
\hline Distance & {$\left[323_{-96}^{+233}, 322_{-122}^{+504}\right] \mathrm{pc} / 357 \pm 52 \mathrm{pc}[2,5]$} \\
\hline Age & $2.39_{-2.05}^{+3.41} \operatorname{Myr}[2$, here $]$ \\
\hline $\mathrm{H}_{\alpha}$ equivalent width & $-11.40 \AA[$ [2] \\
\hline LiI equivalent width & $0.40 \AA[2]$ \\
\hline$v \sin (i)_{*}$ & $80.6 \pm 8.1 \mathrm{~km} \mathrm{~s}^{-1}[3]$ \\
\hline Proper motion [E, N] & {$[-0.1 \pm 5.3,0.9 \pm 5.5] \mathrm{mas} / \mathrm{yr}[6]$} \\
\hline$B, V, R$ photometry & {$[18.35,16.26,15.19] \operatorname{mag}[7,2,3]$} \\
\hline \multirow[t]{2}{*}{$J, H, K$ photometry } & {$[12.232 \pm 0.028,11.559 \pm 0.026,11.357 \pm 0.021] \mathrm{mag}[8]$} \\
\hline & CVSO 30 b/PTFO 8-8695 b \\
\hline (Projected) separation & $0.00838 \pm 0.00072 \mathrm{au}[3]$ \\
\hline Period (circular) & $0.448413 \pm 0.000040 \mathrm{~d}[3]$ \\
\hline Orbit. inclination & $61.8 \pm 3.7^{\circ}[3]$ \\
\hline Orbit. misalignment & $69 \pm 2^{\circ} / 73.1 \pm 0.5^{\circ}[4]$ \\
\hline
\end{tabular}

References. [1] Briceño et al. (2007a); [2] Briceño et al. (2005); [3] van Eyken et al. (2012); [4] Barnes et al. (2013); [5] Downes et al. (2014); [6] Zacharias et al. (2013); [7] Zacharias et al. (2004); [8] Cutri et al. (2003), Skrutskie et al. (2006).

Table 2. CVSO 30 astrometry and photometry.

\begin{tabular}{lcc}
\hline \hline & CVSO 30 b/ & CVSO 30 c \\
& PTFO 8-8695 b & \\
\hline & Separation with respect to the host star [E, N] & {$[175.453,63.395]$ pixel } \\
2010 September 25 & & {$[1.746 \pm 0.006$,} \\
2012 December 3 & & $0.621 \pm 0.010]^{\prime \prime}$ \\
\hline (Projected) separation & $0.00838 \pm 0.00072 \mathrm{au}[1]$ & $662 \pm 96 \mathrm{au}$ \\
Period (circular) & $0.448413 \pm 0.000040 \mathrm{~d}[1]$ & $\sim 27250 \mathrm{yr}$ \\
Orbit. inclination & $61.8 \pm 3.7^{\circ}[1]$ & \\
Orbit. misalignment & $69 \pm 2^{\circ} / 73.1 \pm 0.5^{\circ}[2]$ & \\
\hline$z^{\prime}$-band (differential) & & $>6.8 \mathrm{mag}$ \\
\hline$J$-band (differential) & & $7.385 \pm 0.045 \mathrm{mag}$ \\
$H$-band (differential) & & $7.243 \pm 0.014 \mathrm{mag}$ \\
$K$ s-band (differential) & & $7.351 \pm 0.022 \mathrm{mag}$ \\
\hline$J$-band (differential) & & $7.183 \pm 0.035 \mathrm{mag}$ \\
\hline
\end{tabular}

References. [1] van Eyken et al. (2012); [2] Barnes et al. (2013).

distance of the star. No astrometric calibrator could be found in the night of the Keck observations (hence the position of the object is given in pixels in Table 2), but we note that the position is consistent with the VLT data. We used the nominal pixel scale of NIRC2 of $0.009942^{\prime \prime} /$ pixel $\left( \pm 0.00005^{\prime \prime}\right)$ and assumed $0^{\circ}$ detector orientation for the Keck epoch, which results in 1.744 arcsec right ascension and 0.630 arcsec declination separation in the relative position of CVSO $30 \mathrm{c}$ with respect to its host star. The consistency was expected for a companion because the proper motion of CVSO 30 is too small to distinguish a background source from a sub-stellar companion based on common proper motion (Table 1).

CVSO 30 is in general currently not suitable for a common proper motion analysis because the errors in proper motion exceed the proper motion values (Table 1). As orbital motion around the host star might be detectable, we performed a 
Table 3. VLT/NACO, VLT/SINFONI, archival KeckII/NIRC2, and Calar Alto/2.2 m/AstraLux observation log.

\begin{tabular}{|c|c|c|c|c|c|c|c|c|c|c|}
\hline Instrument & $\begin{array}{l}\text { JD-2 } 455000 \\
\text { [days] }\end{array}$ & $\begin{array}{c}\text { Date of } \\
\text { observation }\end{array}$ & $\begin{array}{c}\text { DIT } \\
{[\mathrm{s}]}\end{array}$ & NDIT & $\begin{array}{c}\# \\
\text { images }\end{array}$ & Airmass & $\begin{array}{l}\text { DIMM }^{a} \\
\text { Seeing }\end{array}$ & $\begin{array}{l}\tau_{0}{ }^{b} \\
{[\mathrm{~ms}]}\end{array}$ & $\begin{array}{c}\text { Strehl } \\
{[\%]}\end{array}$ & $\begin{array}{c}S / N \\
\text { (brightest pixel) }\end{array}$ \\
\hline NACO $J$ & 1264.69416 & 03 Dec. 2012 & 15 & 4 & 15 & 1.13 & 0.8 & 3.7 & 3.2 & 5.9 \\
\hline NACO $H$ & 1264.70764 & 03 Dec. 2012 & 15 & 4 & 15 & 1.12 & 0.6 & 4.6 & 11.2 & 24.6 \\
\hline NACO $K \mathrm{~s}$ & 1264.72079 & 03 Dec. 2012 & 15 & 4 & 15 & 1.11 & 0.7 & 4.6 & 23.7 & 11.1 \\
\hline NACO $J$ & 1266.72899 & 05 Dec. 2012 & 30 & 2 & 15 & 1.12 & 1.3 & 2.8 & 2.0 & 6.6 \\
\hline SINFONI $H+K$ & 1592.82609 & 27 Oct. 2013 & 300 & 2 & 3 & 1.12 & 0.5 & 5.0 & & $\lesssim 15$ \\
\hline NIRC2 $H$ & 465.05374 & 25 Sep. 2010 & 3 & 10 & 12 & 1.25 & 0.4 & & 7.0 & 7.8 \\
\hline AstraLux $z^{\prime}$ & 2260.6696 & 26 Aug. 2015 & 0.02945 & 1 & 70000 & 1.73 & 1.1 & & no $\mathrm{AO}$ & non-detection \\
\hline
\end{tabular}

Notes. Remarks: ${ }^{(a)}$ Differential image motion monitor (DIMM) seeing average of all images; ${ }^{(b)}$ coherence time of atmospheric fluctuations.
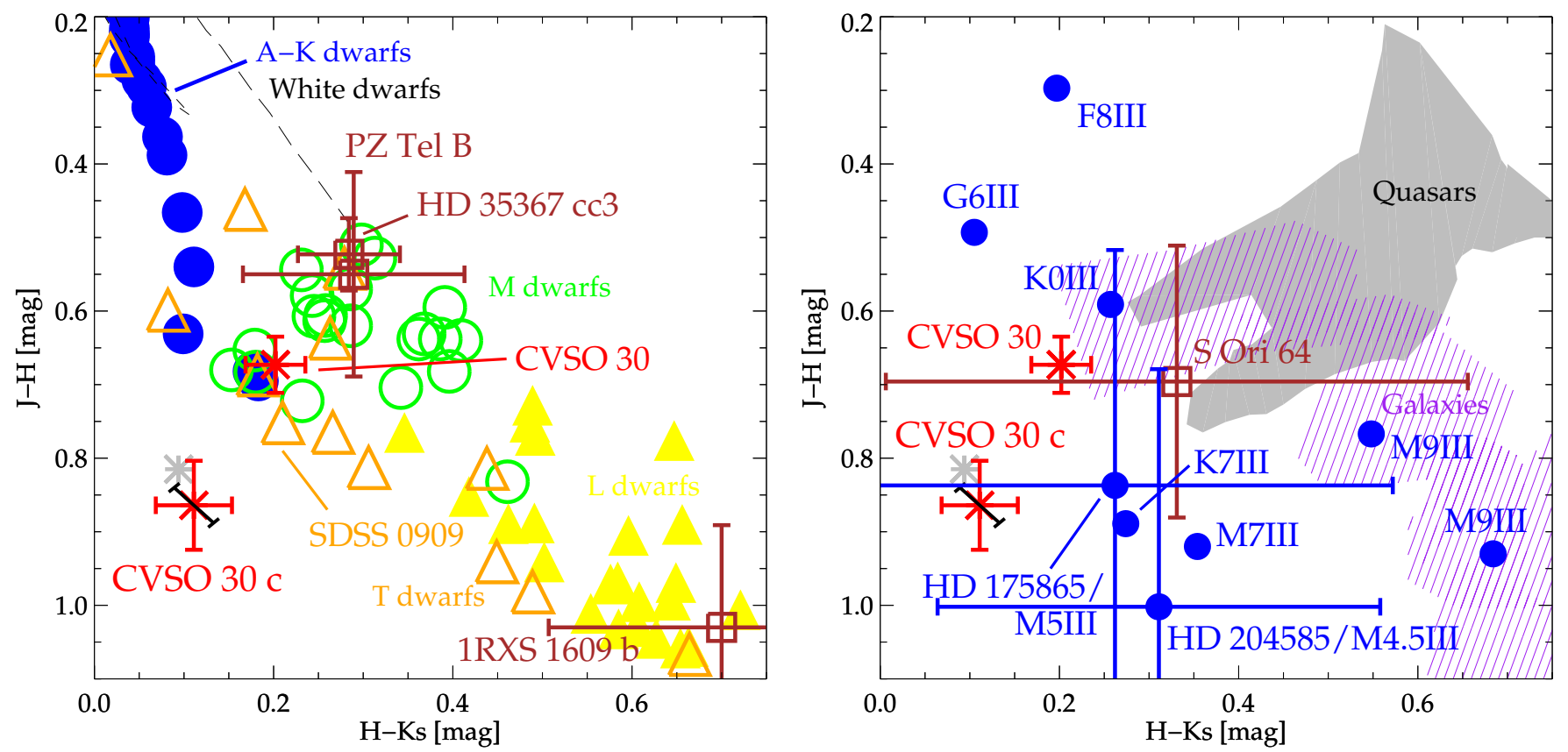

Fig. 2. CVSO 30, CVSO 30 c, and comparison objects, superimposed onto the colour data from Hewett et al. (2006). CVSO $30 \mathrm{c}$ clearly stands out in the lower left corner, approximately consistent with colours of giants, early-M and early- $\mathrm{T}$ dwarfs, and free-floating planetary mass objects (Zapatero Osorio et al. 2000; Peña Ramírez et al. 2012), e.g. consistent with absolute magnitude and $J-K$ s colour of S Ori 64. Its unusual blue colour can most likely be attributed to the youth of these objects (Saumon \& Marley 2008), leading to L-T transition opacity drop at high brightnesses (see Fig. 11). See Fig. A.5 for details. For CVSO $30 \mathrm{c}$ we give the colours before (grey) and after (red) correcting for the NACO to the 2MASS filter set and we give the maximum possible systematic photometric offsets caused by the variability of the primary star that is used as reference (black).

dedicated orbit estimation for the wide companion. The analysis shows that even after two to three years of epoch difference, no significant orbital motion is expected for the wide companion (Fig. A.1).

Using the Two Micron All Sky Survey (2MASS; Cutri et al. 2003; Skrutskie et al. 2006) photometry for the primary and our NACO images for differential brightness measurements, we find CVSO $30 \mathrm{c}$ to exhibit an unusually blue $H-K$ s colour, while its $J-H$ colour indicates the companion candidate to be redder than the primary. This implies that the companion is too red to be an eclipsing background binary mimicking the transiting signal of CVSO 30 as a false-positive signal, which is further indication for the planetary nature of CVSO $30 \mathrm{~b}$.

The differential photometry (Table 2) of CVSO $30 \mathrm{c}$ was achieved using psf fitting with the Starfinder package of IDL (Diolaiti et al. 2000) using the primary star CVSO 30 as psf reference. First the noise of the final jittered image was computed, taking the photon noise, the gain, RON, and the number of combined images into account, and then it was transferred to the starfinder routine for psf fitting. This resulted in the values given in Table 2 . The values were checked with aperture photometry.

As given in van Eyken et al. (2012), our psf reference CVSO 30 varies by $0.17 \mathrm{mag}$ ( $\min$ to $\max$ ) in the $R$-band, consistent with our estimates within YETI. The present steep wavelength dependence of the variability amplitudes is best described by hot star-spots (Koen 2015), therefore we can extrapolate from measurements of the very similar T Tauri GQ Lup (Broeg et al. 2007). According to this, 0.17 mag in $R$ correspond to about $0.1 \mathrm{mag}$ and $0.055 \mathrm{mag}$ variability in $J$ and $K$ s-band, respectively. As the hot spots change the bands simultaneously, this gives rise to a maximum systematic offset of $0.045 \mathrm{mag}$ in $J-K$ s colour. We give an estimate of this variability as black error bars for a possible additional systematic offset of CVSO $30 \mathrm{c}$ in Fig. 2. 
T. O. B. Schmidt et al.: Direct Imaging of a second planet candidate in the transiting CVSO 30 system

Table 4. CVSO 30 deduced planetary properties.

\begin{tabular}{lcc}
\hline \hline & CVSO 30 b/ & CVSO 30 c \\
& PTFO 8-8695 b & \\
\hline Opt. extinction & & $0.19_{-0.19}^{+2.51} \mathrm{mag}$ \\
Luminosity (vs. $\odot$ ) & & $-3.78_{-0.13}^{+0.33} \mathrm{dex}$ \\
Eff. temperature $T_{\text {eff }}$ & & $1600_{-300}^{+120} \mathrm{~K}$ \\
Surface gravity $\log g$ & & $3.6_{-0.6}^{+1.4} \mathrm{dex}$ \\
Radius & $1.91 \pm 0.21 R_{\text {Jup }}[1]$ & $1.63_{-0.34}^{+0.87} R_{\text {Jup }}$ \\
& $1.64 / 1.68 \pm 0.07 R_{\text {Jup }}[2]$ & $4.3_{-3.7}^{+4.9} M_{\text {Jup }}(\log g \&$ Roche $)$ \\
Mass & $<5.5 \pm 1.4 M_{\text {Jup }}[1]$ & $4.7_{-2.0}^{+5.5} M_{\text {Jup }}(L$, age $)$ \\
& $3.0 \pm 0.2 M_{\text {Jup }}[2]$ & $4.7_{-2.0}^{+3.6} M_{\text {Jup }}\left(L, T_{\text {eff }}\right.$, age $)$ \\
& $3.6 \pm 0.3 M_{\text {Jup }}[2]$ & $<10 M_{\text {Jup }}\left(z^{\prime}\right.$ imaging limit $)$
\end{tabular}

References. [1] van Eyken et al. (2012), [2] Barnes et al. (2013).

Table 5. Astrometric calibration of VLT/NACO.

\begin{tabular}{clcc}
\hline \hline Object & $\begin{array}{l}\text { JD }-2456000 \\
{[\text { days }]}\end{array}$ & $\begin{array}{c}\text { Pixel scale } \\
{[\text { mas/pixel }]}\end{array}$ & $\begin{array}{c}\mathrm{PA}^{a} \\
{[\mathrm{deg}]}\end{array}$ \\
\hline 47 Tuc & 264.62525 & $13.265 \pm 0.041$ & $+0.60 \pm 0.31$ \\
\hline
\end{tabular}

Notes. All data from $K$ s-band images. ${ }^{(a)} \mathrm{PA}$ is measured from $\mathrm{N}$ over E to $S$.

The colours of CVSO 30 and CVSO $30 \mathrm{c}$ are very similar (Table 2 and Fig. 2). We currently lack a spectrum of CVSO $30 \mathrm{c}$ in $J$-band, therefore we used the M3V star Gl 388 (Cushing et al. 2005; Rayner et al. 2009) and the L3/L4 brown dwarf 2MASS J11463449+2230527 (Cushing et al. 2005) to derive a preliminary filter correction between 2MASS and NACO for CVSO 30 and CVSO 30 c. The colours of CVSO 30 are well known from 2MASS (Table 1), the differential brightnesses to CVSO 30 c vary from NACO to 2 MASS by 28 mmag in $J$, $-21 \mathrm{mmag}$ in $H$, and $-38 \mathrm{mmag}$ in $K \mathrm{~s}$. Thus CVSO $30 \mathrm{c}$ is 49 mmag redder in $J-H$ and 17 mmag redder in $H-K \mathrm{~s}$ in 2MASS (red in Fig. 2) than in the NACO results (grey in Fig. 2).

In Fig. 2 and Table 6 we compare CVSO $30 \mathrm{c}$ to the colours of several possible sources. We find that background stars of spectral types OBAFGK are too blue in $J-H$, late-M dwarfs are too blue in $J-H$ and too red in $H-K$, while foreground L- and late T-dwarfs are either too red in $H-K$ or too blue in $J-H$. In addition, background galaxies, quasars, and $\mathrm{H} / \mathrm{He}$ white dwarfs are also inconsistent with the values of CVSO $30 \mathrm{c}$. Only late-type giants, early-M and early-T dwarfs, and planetary mass free-floating objects such as are found in the $\sigma$ Orionis star cluster have comparable colours (Zapatero Osorio et al. 2000; Peña Ramírez et al. 2012).

\section{CVSO $30 \mathrm{c}$ spectroscopic analysis}

A common proper motion analysis is not feasible because of the low proper motion of the host star (Table 1), therefore we carried out spectroscopic follow-up observations at the end of 2013, using the ESO VLT integral field unit SINFONI. The observations were made in $H+K$-band with 100 mas/spaxel scale (FoV: $3 \operatorname{arcsec} \times 3 \operatorname{arcsec})$. The instrument provides information in the two spatial directions of the sky in addition to the simultaneous $H$ - and $K$-band spectra. An unfortunate timing of the observations led to a parallactic angle at which a spike, probably of the
Table 6. Photometric rejection significance, spectroscopic reduced $\chi^{2}$ results, and corresponding formal significance without systematics for different comparison objects.

\begin{tabular}{|c|c|c|c|c|c|c|}
\hline \multirow[t]{2}{*}{ Object } & \multirow[t]{2}{*}{$\mathrm{SpT}$} & \multicolumn{2}{|c|}{ Photometry } & \multirow{2}{*}{$\begin{array}{l}\text { add. } \\
\text { Ref. }\end{array}$} & \multicolumn{2}{|c|}{ Spectroscopy } \\
\hline & & $\begin{array}{c}J-H \\
{[\sigma]} \\
\end{array}$ & $\begin{array}{c}H-K \mathrm{~s} \\
{[\sigma]} \\
\end{array}$ & & $\begin{array}{l}H \text {-band } \\
{\left[\sigma / \chi_{r}^{2}\right]}\end{array}$ & $\begin{array}{l}K \text {-band } \\
{\left[\sigma / \chi_{r}^{2}\right]} \\
\end{array}$ \\
\hline HD 237903 & K7V & 3.4 & 0.5 & [1] & $>6 / 2.66$ & $>6 / 1.60$ \\
\hline Gl 846 & MOV & 2.8 & 1.8 & [1] & $>6 / 2.38$ & $5.4 / 1.51$ \\
\hline Gl 229 & M1V & 0.6 & 0.3 & {$[1]$} & $>6 / 2.37$ & $5.3 / 1.50$ \\
\hline Gl 806 & $\mathrm{M} 2 \mathrm{~V}$ & 4.5 & 2.5 & {$[1]$} & $>6 / 2.73$ & $4.3 / 1.40$ \\
\hline Gl 388 & M3V & 3.7 & 2.8 & [2], [1] & $>6 / 2.57$ & $3.7 / 1.33$ \\
\hline Gl 213 & M4V & 5.5 & 2.6 & {$[2],[1]$} & $>6 / 2.80$ & $2.5 / 1.21$ \\
\hline Gl 51 & M5V & 3.8 & 3.5 & [2], [1] & $>6 / 2.47$ & $2.6 / 1.21$ \\
\hline Gl 406 & M6V & 3.4 & 4.6 & [2], [1] & $>6 / 2.50$ & $2.5 / 1.20$ \\
\hline Gl 644C & M7V & 4.1 & 5.1 & [2], [1] & $>6 / 2.87$ & $2.2 / 1.17$ \\
\hline Gl 752B & M8V & 2.6 & 6.4 & [2], [1] & $>6 / 2.76$ & $2.3 / 1.18$ \\
\hline LHS 2065 & M9V & 1.7 & 7.5 & {$[1]$} & $>6 / 2.45$ & $2.2 / 1.17$ \\
\hline LHS 2924 & L0 & 1.4 & 6.5 & [2], [1] & $>6 / 2.77$ & $2.1 / 1.16$ \\
\hline 2MUCD 20581 & L1 & 2.2 & 7.5 & {$[2]$} & $>6 / 3.96$ & $3.7 / 1.33$ \\
\hline Kelu-1AB & $\mathrm{L} 2+\mathrm{L} 3.5$ & 2.2 & 9.8 & [2] & $>6 / 3.68$ & $3.6 / 1.32$ \\
\hline 2MUCD 11291 & L3 & 1.8 & $>10$ & [2] & $>6 / 3.66$ & $3.8 / 1.34$ \\
\hline 2MUCD 12128 & L4.5 & 5.5 & $>12$ & [2] & $>6 / 3.09$ & $3.4 / 1.29$ \\
\hline 2MUCD 11296 & L5.5 & 1.3 & $>10$ & [2] & $>6 / 4.60$ & $5.5 / 1.52$ \\
\hline 2MUCD 11314 & L6 & 2.0 & 8.4 & [2] & $>6 / 3.64$ & $>6 / 1.66$ \\
\hline 2MUCD 10721 & L7.5 & 5.8 & $>11$ & [2] & $>6 / 3.49$ & $3.4 / 1.29$ \\
\hline 2MUCD 10158 & L8.5 & 2.5 & 9.8 & [2] & $>6 / 4.87$ & $5.0 / 1.47$ \\
\hline SDSS $1520+354$ & T0 & 1.0 & 5.4 & {$[3]$} & $>6 / 4.63$ & $>6 / 2.15$ \\
\hline SDSS $0909+652$ & $\mathrm{~T} 1.5$ & 0.3 & 0.4 & [4] & $>6 / 8.04$ & $>6 / 3.64$ \\
\hline SDSS 1254-012 & $\mathrm{T} 2$ & 0.8 & 2.0 & [2] & $>6 / 7.97$ & $>6 / 2.90$ \\
\hline 2MASS 055-140 & $\mathrm{T} 4$ & 9.4 & 0.1 & [2] & $>6 / 16.2$ & $>6 / 19.1$ \\
\hline HD 204585 & M4.5III & 0.4 & 0.8 & [1] & $>6 / 1.86$ & $>6 / 1.88$ \\
\hline HD 175865 & M5III & 0.1 & 0.5 & [1] & $>6 / 1.91$ & $>6 / 1.78$ \\
\hline BK Vir & M7III & 0.4 & 0.9 & [1] & $>6 / 2.47$ & $>6 / 1.72$ \\
\hline HY Aqr & M8-9III & 0.6 & 8.5 & [1] & $>6 / 5.78$ & $>6 / 1.61$ \\
\hline Galaxies & various & 4.2 & 3.0 & [5], [6] & $>6 / 2.28$ & $>6 / 1.61$ \\
\hline Quasars & - & 4.4 & 3.9 & {$[5]$} & - & - \\
\hline White Dwarfs & various & 6.4 & 3.9 & [5] & - & - \\
\hline CVSO 30 & M3 & 2.7 & 1.7 & & $>6 / 3.31$ & $6.0 / 1.57$ \\
\hline PZ Tel B & M7 & 2.1 & 1.4 & [7] & $>6 / 3.29$ & $3.0 / 1.25$ \\
\hline CT Cha b & M9 & 0.4 & 1.3 & [8], [9] & $>6 / 2.27$ & $1.8 / 1.13$ \\
\hline $2 \mathrm{M} 0441 \mathrm{Bb}$ & $\mathrm{L} 1$ & 0.5 & 2.4 & {$[10]$} & $>6 / 3.13$ & $1.9 / 1.13$ \\
\hline 1RXS 1609 b & $\mathrm{L} 4$ & 1.1 & 3.0 & [11] & $>6 / 2.70$ & $2.3 / 1.18$ \\
\hline$\beta$ Pic b & $\mathrm{L} 4$ & 1.0 & 3.5 & [12] & $>6 / 2.06$ & - \\
\hline $2 \mathrm{M} 1207 \mathrm{~b}$ & L7 & 3.5 & 4.4 & [13] & $>6 / 2.66$ & $2.5 / 1.20$ \\
\hline S Ori 64 & $\mathrm{~L} / \mathrm{T}$ & 0.9 & 0.7 & [14] & - & - \\
\hline DP (Fig. 4) & - & - & - & {$[15]$} & $2.2 / 1.16$ & $2.0 / 1.14$ \\
\hline
\end{tabular}

References. [1] Rayner et al. (2009); [2] Cushing et al. (2005); [3] Burgasser et al. (2010a); [4] Chiu et al. (2006); [5] Hewett et al. (2006); [6] Mannucci et al. (2001); [7] Schmidt et al. (2014); [8] Schmidt et al. (2009); [9] Schmidt et al. (2008); [10] Bowler \& Hillenbrand (2015); [11] Lafrenière et al. (2008); [12] Chilcote et al. (2015); [13] Patience et al. (2010); [14] Peña Ramírez et al. (2012); from VISTA to 2MASS magnitudes using colour equations from http://casu.ast.cam.ac.uk/surveys-projects/vista/ technical/photometric-properties; [15] Helling et al. (2008).

telescope secondary mounting, was superimposed onto the wellseparated spectrum of the companion candidate CVSO $30 \mathrm{c}$.

After correcting, the resulting spectrum can be compared to model atmospheres to determine its basic properties and to other sub-stellar companions to assess its youth and the reliability of the models at this low age, surface gravity, and temperature regime.

In an attempt to optimally subtract the spike of the host star, we performed several standard and customised reduction steps. After dark subtraction, flat-fielding, wavelength calibration, and 

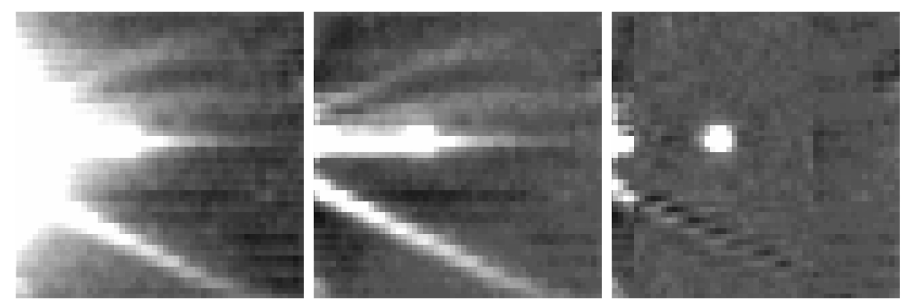

Fig. 3. Median in wavelength direction of the reduced VLT/SINFONI integral field cubes. Left: cube after reduction. Centre: cube after removing the primary halo, assumed to be centred at the separation of $1.85^{\prime \prime}$, as measured in the VLT/NACO images. North is about $70^{\circ}$ from the right-hand side towards the bottom of the plots. Right: cube after removing the primary halo, spectral deconvolution, and polynomial flattening of the resulting background, used for the extraction of the final spectrum.

cube reconstruction, we found that the spike was superimposed onto the companion in every one of the three individual exposures, but at slightly different orientation angles (Fig. 3, left panel). As a first step, we used the NACO astrometry to determine the central position of the primary, which is itself outside the observed field of view of the integral field observations. The orientation of the SINFONI observations was intentionally chosen to leave the connection line of primary and companion exactly in $x$ direction. The primary is about 1.85 arcsec exactly to the left of CVSO $30 \mathrm{c}$ in the data because the $x$ direction offers a twice as good sampling regarding the number of pixels for the separation. We were thus able to subtract the radial symmetric halo of the host star from the data cube (Fig. 3, central panel) using the nominal spatial scale. This is necessary because the halo of the primary star is determined by the AO performance at the different wavelength. At this stage, we extracted a first spectrum by subtracting an average spectrum of the spike, left and right of the companion psf from the superposition of companion and spike. We find the results in Fig. A.2 before (red spectrum) and after (blue spectrum) spike subtraction, which also removes the still-present $\mathrm{OH}$ lines. The horizontal spike in Fig. 3 appears too narrow to the right. This is a projection effect because the rotation of the spike within the three median-combined cubes leads to less overlap on the right-hand side of the cube than on the left-hand side. For this reason, the continuum in Fig. A.2 is not trustworthy because the flux of the spike below the companion candidate is not the average of the spike flux to the left and right of the object.

We tried several methods to remove the spike and decided to follow the spectral deconvolution technique (Sparks \& Ford 2002; Thatte et al. 2007). This method is able to distinguish both the wavelength-dependent airy rings and speckles and the spike from the light of the wavelength-independent companion position by using the long wavelength coverage of the observed data cube. As given in Thatte et al. (2007) for the same instrument, the bifurcation radius for SINFONI $H+K$ is for $\epsilon=1.1 r=246$ mas, and for $\epsilon=1.2 r=268$ mas, which means that parts of the data without contamination of the companion could be found at the much higher separation of about 1.85 arcsec. The reduction was then completed by applying a polynomial background correction around CVSO $30 \mathrm{c}$ because the previous reduction steps left a low spatial frequency remnant around it (Fig. 3, right panel). Finally, the optimal extraction algorithm (Horne 1986) was performed around the companion and subtracted by the corresponding background flux from the close, well-corrected vicinity, and the telluric atmosphere correction using HD 61957, a B3V spectroscopic standard observed in the same night.
We first compare the spectrum of CVSO $30 \mathrm{c}$ to spectra derived from Drift-Phoenix atmosphere simulations. These are dedicated radiative transfer models that take the strong continuum altering influence of dust cloud formation in the detectable parts of planetary atmospheres into account (Helling et al. 2008). From a $\chi^{2}$ comparison of the $H$-and $K$-band spectra to the model grid, we find an effective temperature of about 1800-1900 K, while the individual fit of the $H$ - and $K$-band spectrum give a lower $T_{\text {eff }}$ of about $1600 \mathrm{~K}$. In addition, the slope of the blue part of the triangular $H$-band is too steep in the atmosphere models of about $1800 \mathrm{~K}$ and does not fit the continuum well. The higher $T_{\text {eff }}$ is only needed to fit the unusually blue $H-K$ s colour of the object, as already discussed in the previous photometry section and visible in Fig. 2, since the models do yet not include a good description of the dust opacity drop at the L-T transition. We thus decided to fit the $H$ and $K$-band simultaneously, but normalising them individually, to cope with the unusual colours, while using all the present information for the fit. In this way, we find a best-fitting $T_{\text {eff }}=1600_{-300}^{+120} \mathrm{~K}$, an extinction $A_{V}=0.19_{-0.19}^{+2.51}$ mag, a surface gravity $\log g\left[\mathrm{~cm} / \mathrm{s}^{2}\right]=3.6_{-0.6}^{+1.4} \mathrm{dex}$, and a metallicity $\log \left[(\mathrm{M} / \mathrm{H}) /(\mathrm{M} / \mathrm{H})_{\odot}\right]=0.3_{-0.9}$ dex at the upper supersolar edge of the grid. The $1 \sigma$ fitting contours are shown in Fig. 5, where they delineate the full regime for the error bars, and the best fit itself is shown in Fig. 4.

In Fig. 6 we compare the spectrum of CVSO $30 \mathrm{c}$ to the triangular shaped $H$-band spectrum of the $\beta$ Pic b planet that was obtained with the Gemini Planet Imager (GPI, Chilcote et al. 2015). We also compare this to other planetary mass objects. $\beta$ Pic b is particularly suited as comparison object because it is young (10-20 Myr) and has about the same luminosity and effective temperature (1600-1700 K) while being of higher mass (10-12 $M_{\text {Jup }}$ ). We show linear fits to the blue and red part of the $H$-band and the triangular shape of the chosen Drift-Phoenix models. In contrast to M5 - L5 companions, for which the $\mathrm{H}_{2} \mathrm{O}$ index in Allers et al. (2007) shows an increase in water absorption, the absorption becomes shallower for later spectral types. This means that even though the formal $\chi^{2}$ fit finds a best temperature of $1600 \mathrm{~K}$ for CVSO $30 \mathrm{c}$, the temperature is likely to be lower than for $\beta$ Pic b, exhibiting a steeper $H$-band spectrum. The object's spectrum is not consistent with a giant of any spectral type. The best-fitting giants with consistent photometry (Fig. 2 and Table 6) are shown as comparison in Fig. 6 and would be at a distance of about $200 \mathrm{Mpc}$. To improve the fit in the $K$-band, the spectral type would have to be later than M7III, while the $H$-band does not fit for these objects. Finally, CVSO $30 \mathrm{c}$, which is comparable but younger, must have a lower surface gravity than $\beta$ Pic $\mathrm{b}$, which is determined to have a $1 \sigma$ upper limit of $\log g\left[\mathrm{~cm} / \mathrm{s}^{2}\right]=4.3$ dex according to the linear prior orbit fit in Bonnefoy et al. (2014b). This corrects the surface gravity of CVSO $30 \mathrm{c}$ to $\log g\left[\mathrm{~cm} / \mathrm{s}^{2}\right]=3.6_{-0.6}^{+0.7} \mathrm{dex}$.

\section{AstraLux lucky imaging follow-up observations}

We performed a follow-up of CVSO 30 with 2000 s of AstraLux integration in $z^{\prime}$. The individual AstraLux images were combined using our own pipeline for the reduction of lucky imaging data. The fully reduced AstraLux image is shown in Fig. 7. $z^{\prime}$ photometry of CVSO 30 was not measured so far, but can be derived from its magnitudes in other photometric bands using the colour transformation equations ${ }^{1}$ from Jordi et al. (2006). The $V$ and $R$-band photometry of CVSO 30, as given by Briceño et al. (2005) and van Eyken et al. (2012) $(V=16.26 \pm 0.19$ mag, and

$\overline{1} r-R=0.77 \cdot(V-R)-0.37$ and $r-z^{\prime}=1.584 \cdot(R-I)-0.386$. 


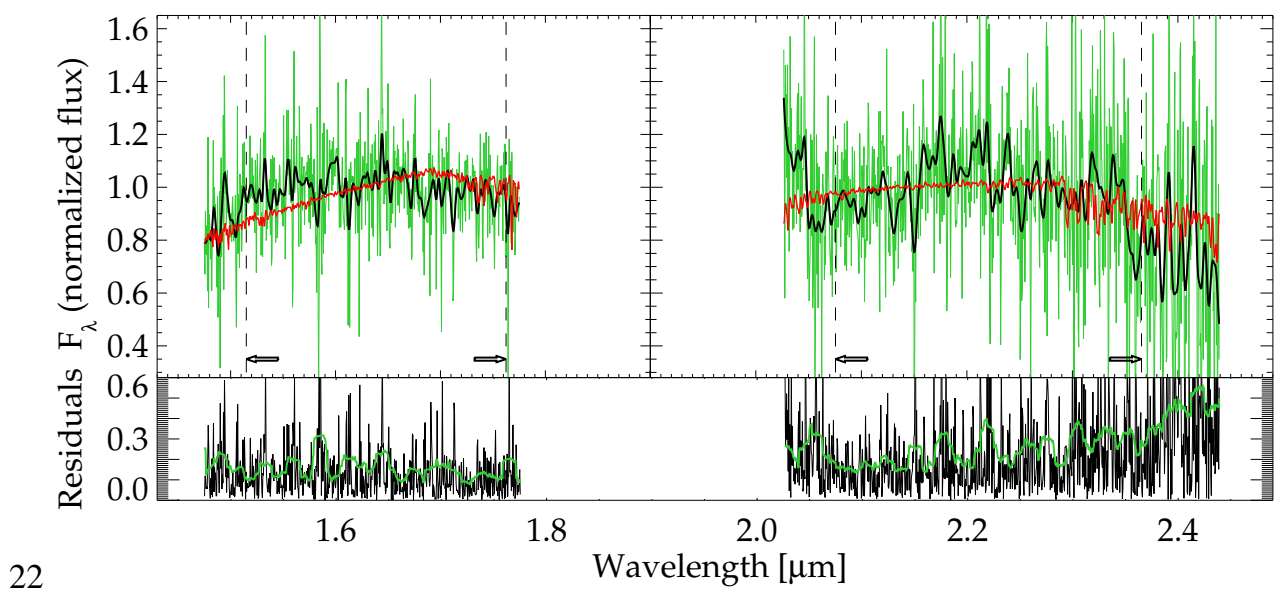

Fig. 4. Spectrum of CVSO $30 \mathrm{c}$ as extracted from the spectral deconvolution-corrected cube in the right panel of Fig. 3. Top: the spectrum in resolution 700 (black) is shown after binning of the original extracted spectrum in resolution 1500 (green). The best-fitting Drift-Phoenix model of Helling et al. (2008) is shown in red, fitting both the individually normalised $H$ and $K$ spectra. This type of normalisation was necessary because the redder colour of the models, in comparison to the unusually blue nature of CVSO $30 \mathrm{c}$, would steer the best-fitting model to higher temperatures, which would prevent fitting the individual features in $H$ and $K$-band. The best-fitting model (red) corresponds to $1600 \mathrm{~K}$, surface gravity $\log g 3.6$ dex, metallicity $[\mathrm{M} / \mathrm{H}] 0.3 \mathrm{dex}$, and 0.19 mag of visual extinction. Bottom: absolute value of the difference between spectrum and model from the top panel (black) versus noise floor at the corresponding position (green).
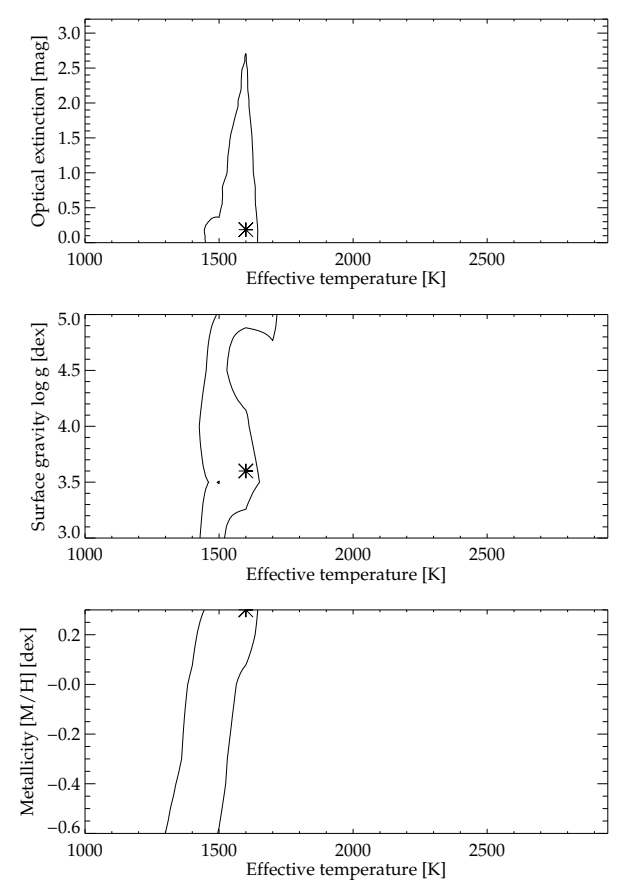

Fig. 5. $1 \sigma$ contour plots of the $\chi^{2}$ Drift-Phoenix model fit to the spectrum shown in Fig. 4. Contour plot in extinction vs. effective temperature (top), surface gravity $\log g$ vs. effective temperature (centre) and metallicity $[\mathrm{M} / \mathrm{H}]$ vs. effective temperature (bottom). The fit shows a best fit at $1600 \mathrm{~K}$, low extinction of $0.19 \mathrm{mag}$, higher values becoming increasingly less likely, and a best fit at $\log g$ 3.6. While all surface gravities seem to be almost of equal probability, a high surface gravity foreground brown dwarf can be excluded from the shape of the $H$-band in Fig. 6. Although the young planetary models differ in photometric colours, this could be because of a not yet fully understood change in the cloud properties at the L-T transition that is indicated by the change in brightness of the L-T transition with age of the system, which we show in Fig. 11.

$R=15.19 \pm 0.085 \mathrm{mag})$, and the $I$-band photometry of the star, listed in the 2005 DENIS database $(I=13.695 \pm 0.030 \mathrm{mag})$, yield $z^{\prime}=13.66 \mathrm{mag}$.
The $(S / N=5)$ detection limit reached in the AstraLux observation is given in Fig. 8. At an angular separation of about 1.8 arcsec from CVSO 30 (or $\sim 640$ au of projected separation), companions that are $\Delta z^{\prime}=6.8$ mag fainter than the star are still detectable at $S / N=5$. The reached detection limit at this angular separation is $z^{\prime}=20.5 \mathrm{mag}$, which is just a tenth of magnitude above the limiting magnitude in the background-noise-limited region around the star at angular separations larger than 2 arcsec. This results in a limiting absolute magnitude of $M_{z^{\prime}}=12.7 \mathrm{mag}$, allowing the detection of sub-stellar companions of the star with masses down to $10 M_{\mathrm{Jup}}$ according to the evolutionary models of Baraffe et al. (2015).

Furthermore, the AstraLux observations also exclude all young ( $3 \mathrm{Myr}$ ) stellar objects (mass higher than $75 M_{\text {Jup }}$ ) that are unrelated to CVSO 30, which are located in the AstraLux field of view at distances closer than about 3410 pc. All young M dwarfs with an age of $3 \mathrm{Myr}$ and masses above $15 M_{\text {Jup }}\left(T_{\text {eff }}>2400 \mathrm{~K}\right)$ can be ruled out up to $530 \mathrm{pc}$. All old stellar objects (mass higher than $75 M_{\text {Jup }}$ ) with an age of 5 Gyr can be excluded when they are located closer than about $130 \mathrm{pc}$.

The AstraLux upper limit results in $z^{\prime}-K \mathrm{~s} \gtrsim 1.75 \mathrm{mag}$, which corresponds to excluding $\gtrsim 0.2 M_{\odot}$ or $\gtrsim 3300 \mathrm{~K}$ (Baraffe et al. 2015) as possible sources or about earlier than M4.5V in spectal type (Kenyon \& Hartmann 1995). Because any object later than $\sim \mathrm{M} 2 \mathrm{~V} / \mathrm{M} 3 \mathrm{~V}$ can be excluded by $\gtrsim 4 \sigma$ from $\mathrm{JHKs}$ photometry (Table 6), no $\mathrm{M}$ dwarf can be a false positive of the new companion candidate CVSO $30 \mathrm{c}$.

\section{Mass determination and conclusions}

With the object brightness determined from the direct near-IR imaging and the information provided by the spectroscopic analysis, we can directly estimate the basic parameters of CVSO 30 c. To determine the luminosity, we considered the extinction law by Rieke \& Lebofsky (1985), a bolometric correction of $\mathrm{BC}_{K}=$ $3.3_{-0.7}^{+0.0}$ mag for spectral type L5-T4 (Golimowski et al. 2004), and a distance of $357 \pm 52$ pc to the 25 Orionis cluster. From the 2MASS brightness of the primary and the differential brightness measured in our VLT NACO data (Table 2) and the extinction value towards the companion derived from spectroscopy, we find 


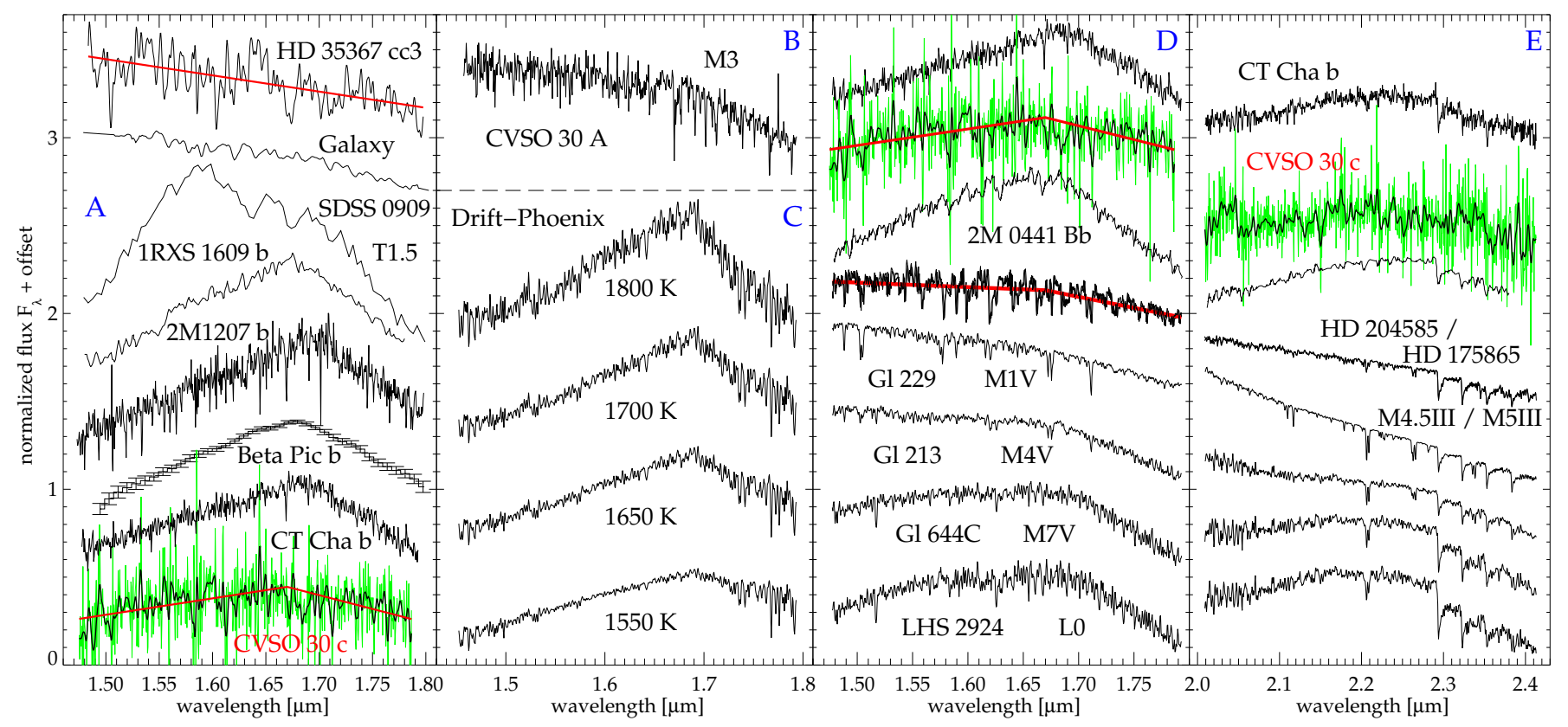

Fig. 6. $H$-band spectrum of CVSO $30 \mathrm{c}$ (lower left) compared to several known planetary candidates and background objects (subplots A, D, $\mathrm{E}$ ). The triangular shape of the $H$-band (A), with red linear fits guiding the eye, indicates that it is not a background galaxy, but a sub-stellar companion. Beta Pic b has approximately the same luminosity and temperature (Chilcote et al. 2015) but a different surface gravity, hence about twice the mass of CVSO $30 \mathrm{c}$. As shown (C), the Drift-Phoenix models indicate that the $H$-band becomes less steep with temperature. This means that CVSO $30 \mathrm{c}$ is even slightly lower in temperature than $\beta$ Pic b. In the upper left panel another candidate is shown, detected at $4.3^{\prime \prime}$ from the A1 star HD 35367, which is about 0.5 mag brighter in the $K$-band than CVSO $30 \mathrm{c}$, but is obviously located in the background. In addition, the $H$-band (D) and $K$-band (E) of CT Cha b and $2 \mathrm{M} 0441 \mathrm{Bb}$, the best-fitting comparison objects, are given in $K$-band. These two and CVSO 30 c are given in $(\mathrm{D}, \mathrm{E})$ with identical offsets in $H$-band and $K$-band. Additionally, the best-fitting giants and a sample of late-type dwarfs is shown for comparison. References and individual reduced $\chi_{r}^{2}$ comparison values are given in Table 6 . Low-resolution spectra of free-floating planetary candidates are not shown, but can be found in Martín et al. (2001).

$\log L_{\mathrm{bol}} / L_{\odot}=-3.78_{-0.13}^{+0.33}$ dex. From the luminosity and effective temperature, we calculate the radius to be $R=1.63_{-0.34}^{+0.87} R_{\mathrm{Jup}}$. In combination with the derived surface gravity, this would correspond to a mass of $M=4.3 M_{\text {Jup }}$, dominated in its errors by high distance and surface gravity uncertainties. While the latter value and the photometry (Fig. 2) would be consistent with a high surface gravity, thus old foreground T-type brown dwarf, but inconsistent with an L-type brown dwarf, the available spectroscopy excludes an old T-type brown dwarf (Fig. 6 and Table $6)$. While the photometry is also consistent with early-M dwarfs, the $K$-band spectroscopy and $z^{\prime}$ upper limit show the opposite behaviour, being only consistent with late-M dwarfs, excluding all types with high significance. Similarly, the remaining $H$-band spectroscopy excludes all comparison objects. Only the bestfitting Drift-Phoenix model (Fig. 4) shows low deviation in $\mathrm{H}$ band, consistent with the fact that the only available very young directly imaged planet candidates exhibit higher temperatures, thus a steeper $H$-band (Fig. 6).

Although recent observations by $\mathrm{Yu}$ et al. (2015) cast doubts on the existence of the inner transiting planet candidate CVSO 30 b or PTFO 8-8695 b, we assume its existence throughout the remaining discussion because all five hypotheses have difficulties in reproducing the observations presented in Yu et al. (2015), including for example different types of starspots. More recently Raetz et al. (2016) and Johns-Krull et al. (2016) presented further evidence for the planetary nature. The inner. The inner planet hypothesis gives another constraint, namely that the system has to be stable with both its planets. As described in van Eyken et al. (2012), CVSO 30 b is very close to its Roche radius, the radius of stability. Assuming the values for mass of

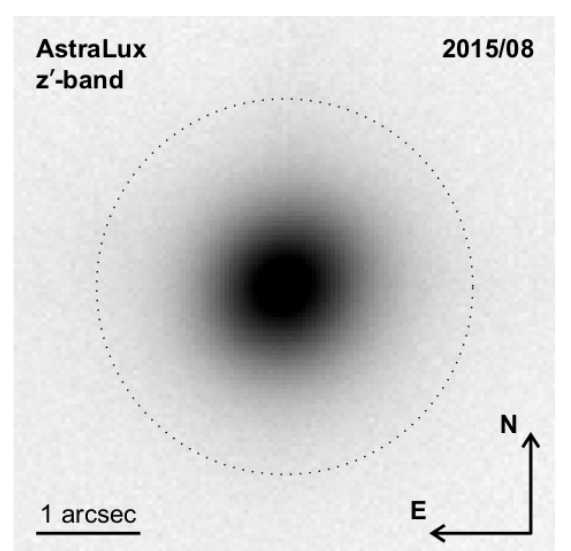

Fig. 7. AstraLux $z^{\prime}$-band image of CVSO 30, taken on Aug 27, 2015. The dotted circle indicates an angular separation of 1.8 arcsec to CVSO 30 (see Fig. 8). No other objects are detected except for the star, which is located in the centre of the AstraLux image.

CVSO $30 \mathrm{~b}$, its radius and orbital period (Tables 2 and 4), we find from the Roche limit an upper limit for the mass of CVSO 30 of $\leq 0.92 M_{\odot}$ for a stable inner system comprised of CVSO $30 \mathrm{~A}$ and b. This mass limit for CVSO 30 is fulfilled at $1 \mathrm{Myr}$ for masses of CVSO $30 \mathrm{c}$ of $\leq 6.9 M_{\text {Jup }}$ at $\leq 760 \mathrm{pc}$ up to $5.8 \mathrm{Myr}$ with masses of CVSO $30 \mathrm{c}$ of $\leq 9.2 M_{\text {Jup }}$ at $\leq 455 \mathrm{pc}$, according to BT-Settl evolutionary models (Allard 2014; Baraffe et al. 2015). Higher ages are not consistent with the age estimate of the primary, but even at $20 \mathrm{Myr}$ we find a mass of CVSO $30 \mathrm{c}$ of $\leq 12.1 M_{\text {Jup }}$ at $\leq 340 \mathrm{pc}$. With the Roche stability criterion for 
T. O. B. Schmidt et al.: Direct Imaging of a second planet candidate in the transiting CVSO 30 system

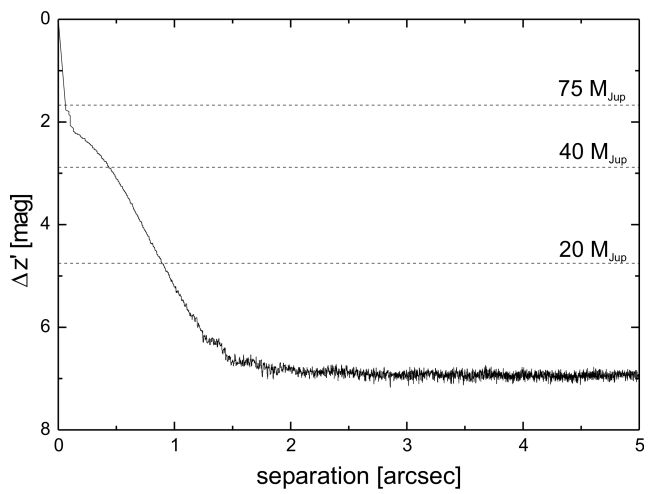

Fig. 8. $S / N=5$ detection limit of our AstraLux observation of CVSO 30 (Fig. 7). The reached magnitude difference that is dependent on the angular separation to the star is shown. The horizontal dashed lines indicate the expected magnitude differences of sub-stellar companions of the star at an age of 3 Myr. Beyond about 1.8 arcsec (or $\sim 640$ au of projected separation), all companions with masses down to $10 M_{\text {Jup }}$ can be excluded around CVSO 30.

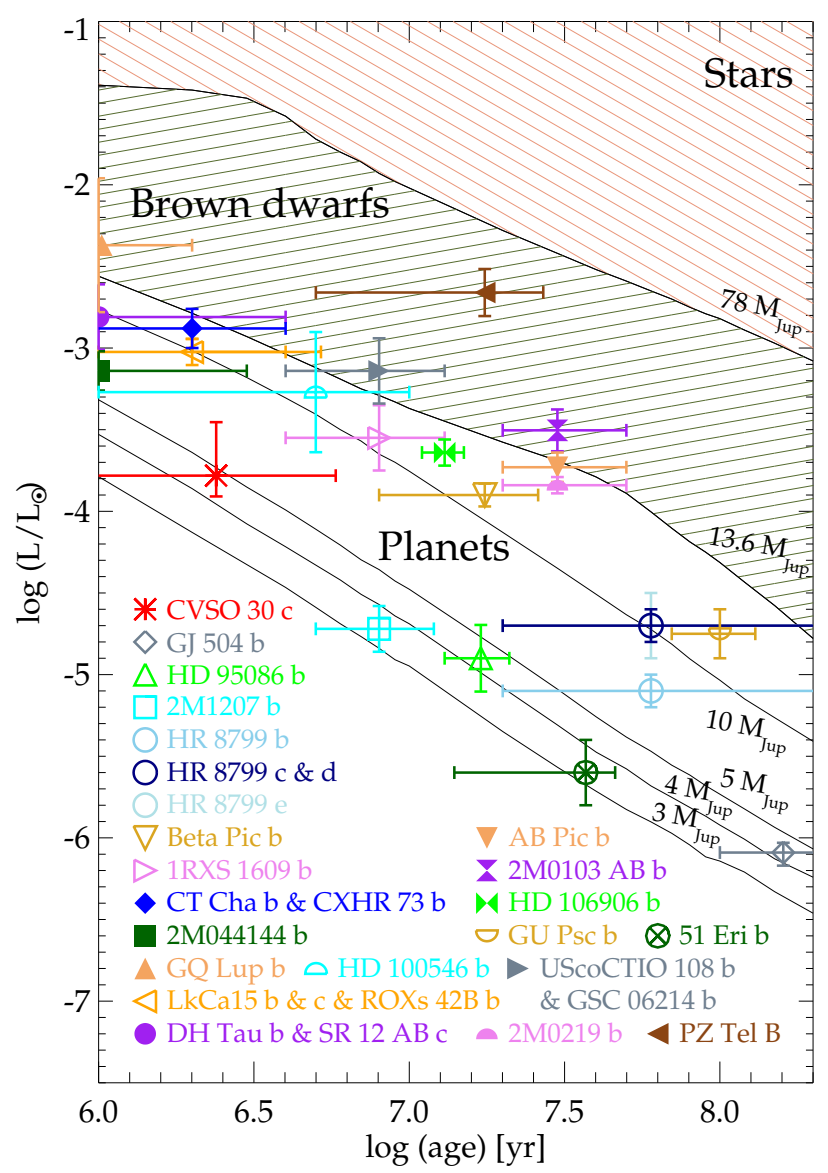

Fig. 9. Evolution of young stars, brown dwarfs, and planets with BTSettl evolutionary tracks (Allard 2014; Baraffe et al. 2015). Shown are a few of the planet candidates known so far in comparison to the new sub-stellar companion candidate CVSO 30 c (see Table A.1).

CVSO $30 \mathrm{~b}$, the previous calculations result in a mass estimate of $M=4.3_{-3.7}^{+4.9} M_{\text {Jup }}$ for CVSO $30 \mathrm{c}$.

For the approximate age of CVSO 30, 2-3 Myr BT-Settl evolutionary models (Allard 2014; Baraffe et al. 2015) predict an apparent brightness of $m_{K} \sim 18.5 \mathrm{mag}$ (assuming the distance to 25 Ori), effective temperature $\sim 1575 \mathrm{~K}$, mass $4-5 M_{\text {Jup }}$, and

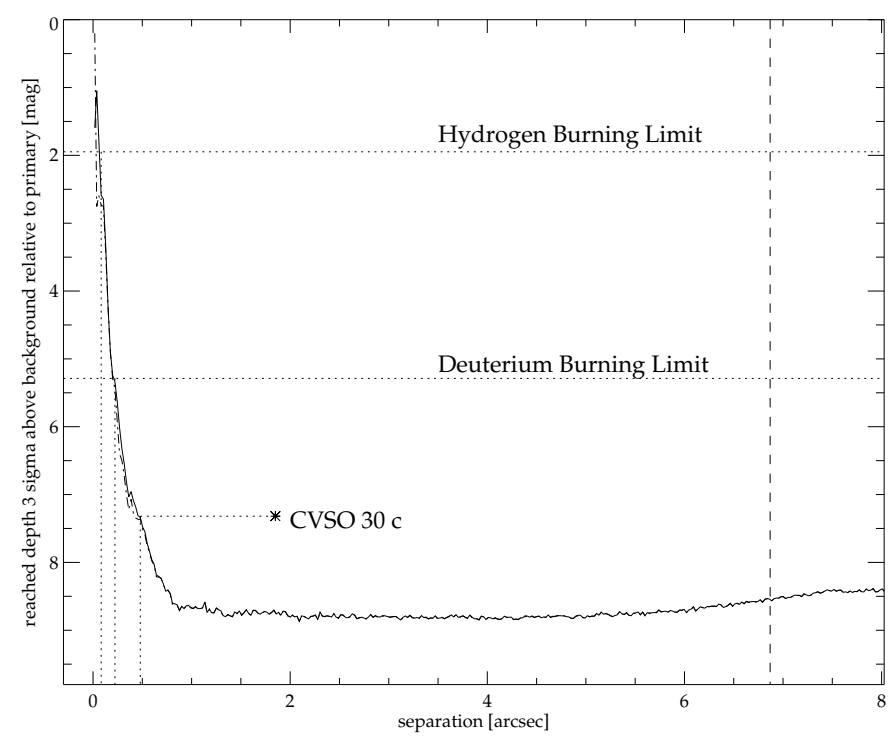

Fig. 10. Dynamic range per pixel achieved in our VLT/NACO $K$ s-band observations, given as $3 \sigma$ contrast to the primary star. The companion would have been detectable until 0.48 arcsec or 171 au separation. A depth of 20.2 mag was reached at maximum, corresponding to $2.8 M_{\text {Jup }}$.

$\log L_{\mathrm{bol}} / L_{\odot} \sim-3.8 \mathrm{dex}$. These expected values are very close to the best-fit atmospheric model spectra fits above, and even the derived visual extinction of about $0.19 \mathrm{mag}$ is very close to the value of the primary $\sim 0.12 \mathrm{mag}$ (Briceño et al. 2005).

Of course, these evolutionary models can also be used to determine the resulting mass, or as mass depends on initial entropy assumptions rather a lower limit (Marleau \& Cumming 2014), from the luminosity and age of the companion candidate and system, respectively. To put CVSO $30 \mathrm{c}$ into context, we show the models and several of the currently known directly imaged planet candidates in Fig. 9. The new companion is one of the youngest and lowest mass companions, and we find a mass of $4.7_{-2.0}^{+5.5} M_{\text {Jup }}$ because the luminosity is not very precise as a result of the rather scarce knowledge of the distance of the system. However, if we take temperature additionally into account, we find a more precise mass determination of $4.7_{-2.0}^{+3.6} M_{\text {Jup }}$, which places CVSO $30 \mathrm{c}$ well within the planetary regime and would mean that it is very close in mass to the probable inner companion of the system CVSO $30 \mathrm{~b}$ with about 2.8-6.9 $M_{\text {Jup }}$ (van Eyken et al. 2012; Barnes et al. 2013).

In Fig. 10 we show the depth reached per pixel in the $K \mathrm{~s}$ band epoch of $20.2 \mathrm{mag}$, corresponding to $2.8 M_{\text {Jup }}$ at the age of CVSO 30, using the same models as above. Brown dwarfs could be found from 30 au outwards, planets from 79 au outwards, and CVSO 30 c could have been found from 171 au outwards.

The core-accretion model (Safronov \& Zvjagina 1969; Goldreich \& Ward 1973; Pollack et al. 1996), one of the much debated planet formation scenarios, is unlikely to form an object in situ at $\geq 662$ au because the timescale would be prohibitively long at such separations. In principle, the object could also have formed in a star-like fashion by turbulent core fragmentation as in the case of a binary star system, since the opacity limit for fragmentation is a few Jupiter masses (Bate 2009), but the large separation and high mass ratio argue against this hypothesis.

The even more obvious possibility would be planet-planet scattering because an inner planet candidate CVSO $30 \mathrm{~b}$ of comparable mass is present that could have been scattered inward at the very same scattering event. Several authors simulated such 


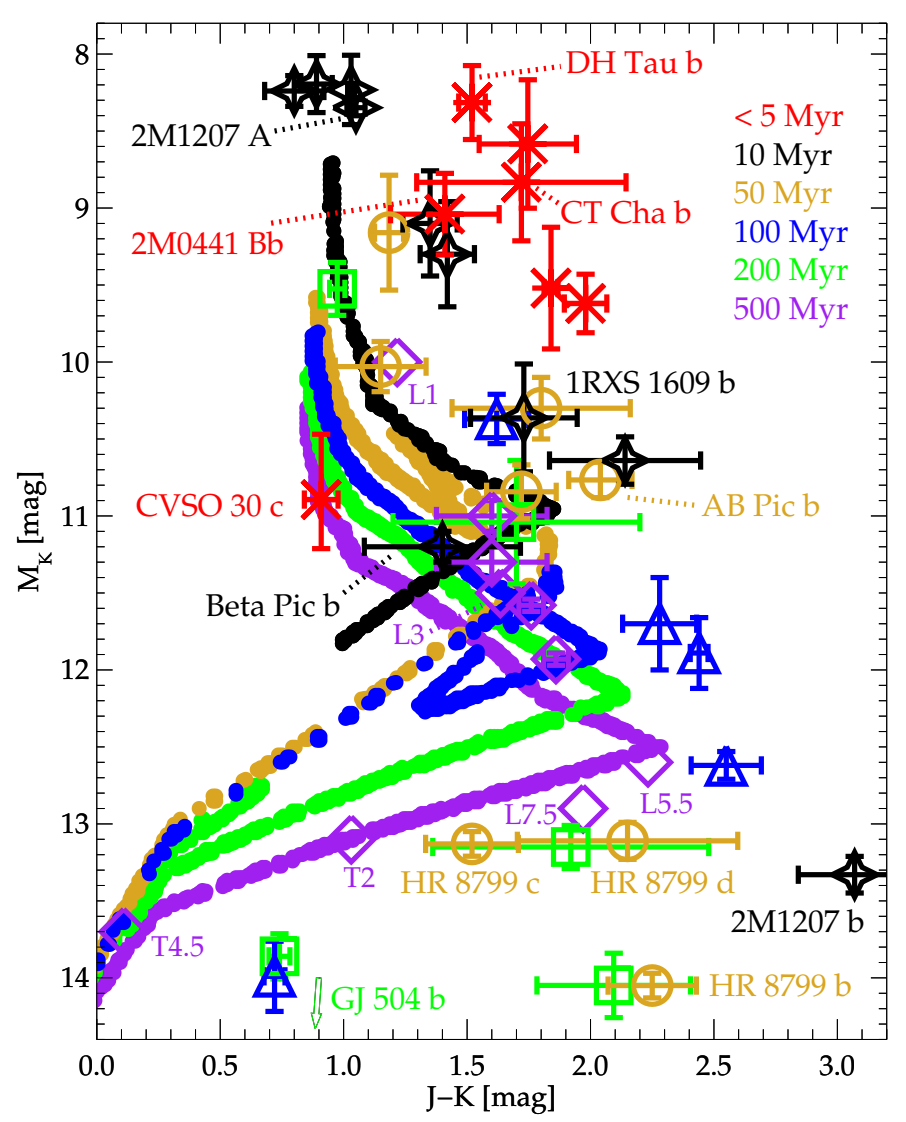

Fig. 11. Colour-magnitude diagram of a simulated cluster brown dwarf population from Saumon \& Marley (2008). Each sequence corresponds to a different age as given in the legend. Superimposed we show the positions of several planet candidates and CVSO $30 \mathrm{c}$. Its unusual blue colour can most likely be attributed to its youth; it is about $2.4 \mathrm{Myr}$ old. The younger the objects, the brighter they are because of not-yetoccurred contraction. Hence they reach the L- and T-dwarf regime at higher brightnesses. If this extrapolation is correct, CVSO $30 \mathrm{c}$ is at the L-T transition, which is roughly consistent with its low effective temperature results. See the discussion and Table A.2 and Fig. A.5 for details.

events and found mostly highly eccentric orbits for the outer scattered planets of up to 100 s or 1000 s of au (Stamatellos \& Whitworth 2009; Nagasawa \& Ida 2011), which is similar to the minimum separation of our outer planet candidate of $662 \mathrm{au}$. The closest match to CVSO $30 \mathrm{bc}$ of a model simulation was presented by Nagasawa \& Ida (2011) with an object at $\sim 300$ au, which has an inner hot planet with which it was scattered. Scattering or gravitational interaction might not be that uncommon as $72 \% \pm 16 \%$ of hot Jupiters are part of multi-planet and/or multi-star systems (Ngo et al. 2015).

The luminosity of CVSO $30 \mathrm{c}$ is only consistent with hotstart models that usually represent the objects formed by gravitational disk-instability, not with cold-start models that are attributed to core-accretion-formed planets (Marley et al. 2007). However, as stated in Spiegel \& Burrows (2012), first-principle calculations cannot yet specify the initial (post-formation) entropies of objects with certainty in the different formation scenarios, hence CVSO $30 \mathrm{c}$ could have formed through a gravitational disk-instability or core accretion and might have been scattered with CVSO $30 \mathrm{~b}$ afterwards.
In this context, it would also be important to clarify the nature of the unusually blue $H-K$ s colour of CVSO $30 \mathrm{c}$. It is consistent with colours of free-floating planets (Fig. 2) and might be caused by its youth, allowing the companion to be very bright, still already being at the L-T transition, which would be consistent with simulations of cluster brown dwarfs at very young ages and their colours in Saumon \& Marley (2008; Fig. 11). This would imply a temperature at the lower end of the $1 \sigma$ errors found for CVSO $30 \mathrm{c}, \leq 1400 \mathrm{~K}$, which is consistent with the less steep $H$-band in comparison to $\beta$ Pic b of about 1600 $1700 \mathrm{~K}$ (Chilcote et al. 2015), however, as shown in Fig. 6. For old brown dwarfs the L-T transition occurs at $T_{\text {eff }} 1200-1400 \mathrm{~K}$, when methane absorption bands start to be ubiquitously seen. However, in the $~ 30$ Myr old planet candidates around HR 8799 no strong methane is found, while the spectrum of the $\sim 90 \mathrm{Myr}$ old object around GU Psc shows strong methane absorption (Naud et al. 2014) all at temperatures of about 1000-1100 K. Thus the L-T transition might be gravity dependent (Marley et al. 2012). Binarity of CVSO 30 c cannot be excluded either, which would also explain the unusual blue $H-K$ s colour.

Since we cannot confirm that CVSO $30 \mathrm{c}$ is co-moving with its host star from our proper motion analysis, we cannot exclude the possibility that CVSO $30 \mathrm{c}$ is a free-floating young planet belonging to the 25 Ori cluster, which is not gravitationally bound to CVSO 30. However, such a coincidence is highly improbable. Zapatero Osorio et al. (2000) searched $847 \operatorname{arcmin}^{2}$ of the $\sigma$ Orionis star cluster for free-floating planets and found only six candidates in the survey with similar colours as CVSO $30 \mathrm{c}$. This means that the probability to find a free-floating planet by chance within a radius of $1.85^{\prime \prime}$ around the transiting planet host star CVSO 30 is about $2 \times 10^{-5}$.

With a mass ratio of planet candidate to star $q=0.0115 \pm$ 0.0015 , CVSO $30 \mathrm{c}$ (and CVSO $30 \mathrm{~b}$ ) is among the imaged planets with the lowest mass ratio (see e.g. De Rosa et al. 2014).

In summary, CVSO $30 \mathrm{~b}$ and $\mathrm{c}$ for the first time allow a comprehensive study of both a transiting and a directly imaged planet candidate within the same system, hence at the same age and even similar masses, using RV, transit photometry, direct imaging, and spectroscopy. Within a few years, the GAIA satellite mission (Perryman 2005) will provide the distance to the system to a precision of about $10 \mathrm{pc}$, which will additionally restrict the mass of CVSO $30 \mathrm{c}$. Simulations of a possible scattering event will profit from the current (end) conditions found for the system. Considering that the inner planet is very close to the Roche stability limit and the outer planet is far away from its host star, the future evolution and stability of the system is also very interesting for dedicated modelling. To investigate how often these scattering events occur, inner planets need also be searched for around other stars with directly imaged wide planets.

Acknowledgements. We thank the ESO and CAHA staff for support, especially during service-mode observations. Moreover, we would like to thank Jeff Chilcote and David Lafrenière for kindly providing electronic versions of comparison spectra from their publications and the anonymous referee, the editor and our language editor for helpful comments that improved this manuscript. TOBS and JHMMS acknowledge support by the DFG Graduiertenkolleg 1351 "Extrasolar Planets and their Host Stars". R.N. and S.R. would like to thank the DFG for support in the Priority Programme SPP 1385 on the "First Ten Million Years of the Solar system" in project NE 515/33-1. S.R. is currently a Research Fellow at ESA/ESTEC. N.V. acknowledges support by fund DIUV38/2011 of Universidad de Valparaiso and by Centro de Astrofísica de Valparaíso (CAV). M.M. wants to thank the German science foundation (DFG) for support in grant MU2695/23-1. This publication makes use of data products from the Two Micron All Sky Survey, which is a joint project of the University of Massachusetts and the Infrared Processing and Analysis Center/California Institute of Technology, funded by the National Aeronautics 
T. O. B. Schmidt et al.: Direct Imaging of a second planet candidate in the transiting CVSO 30 system

and Space Administration and the National Science Foundation. This research has made use of the VizieR catalog access tool and the Simbad database, both operated at the Observatoire Strasbourg. This research has made use of NASA's Astrophysics Data System.

\section{References}

Adams, F. C., \& Laughlin, G. 2001, Icarus, 150, 151

Allard, F. 2014, in IAU Symp. 299, eds. M. Booth, B. C. Matthews, \& J. R. Graham, 271

Aller, K. M., Kraus, A. L., Liu, M. C., et al. 2013, ApJ, 773, 63

Allers, K. N., Jaffe, D. T., Luhman, K. L., et al. 2007, ApJ, 657, 511

Andrews, S. M., Rosenfeld, K. A., Kraus, A. L., \& Wilner, D. J. 2013, ApJ, 771 129

Artigau, É., Gagné, J., Faherty, J., et al. 2015, ApJ, 806, 254

Baglin, A., Auvergne, M., Barge, P., et al. 2007, in Fifty Years of Romanian Astrophysics, eds. C. Dumitrache, N. A. Popescu, M. D. Suran, \& V. Mioc, AIP Conf. Ser., 895, 201

Bailey, V., Meshkat, T., Reiter, M., et al. 2014, ApJ, 780, L4

Baraffe, I., Homeier, D., Allard, F., \& Chabrier, G. 2015, A\&A, 577, A42

Barnes, J. W., van Eyken, J. C., Jackson, B. K., Ciardi, D. R., \& Fortney, J. J. 2013, ApJ, 774, 53

Bate, M. R. 2009, MNRAS, 392, 590

Béjar, V. J. S., Zapatero Osorio, M. R., Pérez-Garrido, A., et al. 2008, ApJ, 673, L185

Biller, B. A., Liu, M. C., Wahhaj, Z., et al. 2010, ApJ, 720, L82

Binks, A. S., \& Jeffries, R. D. 2014, MNRAS, 438, L11

Bonavita, M., Daemgen, S., Desidera, S., et al. 2014, ApJ, 791, L40

Bonnefoy, M., Boccaletti, A., Lagrange, A.-M., et al. 2013, A\&A, 555, A107

Bonnefoy, M., Chauvin, G., Lagrange, A.-M., et al. 2014a, A\&A, 562, A127

Bonnefoy, M., Marleau, G.-D., Galicher, R., et al. 2014b, A\&A, 567, L9

Borucki, W. J., Koch, D., Basri, G., et al. 2010, Science, 327, 977

Boss, A. P. 1997, Science, 276, 1836

Boss, A. P. 2006, ApJ, 637, L137

Bowler, B. P., \& Hillenbrand, L. A. 2015, ApJ, 811, L30

Bowler, B. P., Liu, M. C., Shkolnik, E. L., \& Dupuy, T. J. 2013, ApJ, 774, 55

Bowler, B. P., Liu, M. C., Shkolnik, E. L., \& Tamura, M. 2015, ApJS, 216, 7

Briceño, C., Calvet, N., Hernández, J., et al. 2005, AJ, 129, 907

Briceño, C., Hartmann, L., Hernández, J., et al. 2007a, ApJ, 661, 1119

Briceño, C., Preibisch, T., Sherry, W. H., et al. 2007b, Protostars and Planets V, 345

Broeg, C., Schmidt, T. O. B., Guenther, E., et al. 2007, A\&A, 468, 1039

Burgasser, A. J., Cruz, K. L., Cushing, M., et al. 2010a, ApJ, 710, 1142

Burgasser, A. J., Simcoe, R. A., Bochanski, J. J., et al. 2010b, ApJ, 725, 1405

Burningham, B., Leggett, S. K., Homeier, D., et al. 2011, MNRAS, 414, 3590

Cameron, A. G. W. 1978, Moon and Planets, 18, 5

Carson, J., Thalmann, C., Janson, M., et al. 2013, ApJ, 763, L32

Charbonneau, D., Brown, T. M., Latham, D. W., \& Mayor, M. 2000, ApJ, 529, L45

Chauvin, G., Lagrange, A.-M., Dumas, C., et al. 2004, A\&A, 425, L29

Chauvin, G., Lagrange, A.-M., Dumas, C., et al. 2005a, A\&A, 438, L25

Chauvin, G., Lagrange, A.-M., Lacombe, F., et al. 2005b, A\&A, 430, 1027

Chauvin, G., Lagrange, A.-M., Zuckerman, B., et al. 2005c, A\&A, 438, L29

Chilcote, J., Barman, T., Fitzgerald, M. P., et al. 2015, ApJ, 798, L3

Chiu, K., Fan, X., Leggett, S. K., et al. 2006, AJ, 131, 2722

Close, L. 2010, Nature, 468, 1048

Close, L. M., Siegler, N., Freed, M., \& Biller, B. 2003, ApJ, 587, 407

Close, L. M., Zuckerman, B., Song, I., et al. 2007, ApJ, 660, 1492

Currie, T., Burrows, A., \& Daemgen, S. 2014, ApJ, 787, 104

Cushing, M. C., Rayner, J. T., \& Vacca, W. D. 2005, ApJ, 623, 1115

Cutri, R. M., Skrutskie, M. F., van Dyk, S., et al. 2003, 2MASS All Sky Catalog of point sources

De Rosa, R. J., Patience, J., Ward-Duong, K., et al. 2014, MNRAS, 445, 3694

Delorme, P., Gagné, J., Girard, J. H., et al. 2013, A\&A, 553, L5

Diolaiti, E., Bendinelli, O., Bonaccini, D., et al. 2000, in SPIE Conf. Ser. 4007, ed. P. L. Wizinowich, 879

Downes, J. J., Briceño, C., Mateu, C., et al. 2014, MNRAS, 444, 1793

Ducourant, C., Teixeira, R., Chauvin, G., et al. 2008, A\&A, 477, L1

Dupuy, T. J., Liu, M. C., \& Ireland, M. J. 2014, ApJ, 790, 133

Errmann, R., Raetz, S., Kitze, M., Neuhäuser, R., \& YETI Team 2014, Contributions of the Astronomical Observatory Skalnate Pleso, 43, 513

Faherty, J. K., Rice, E. L., Cruz, K. L., Mamajek, E. E., \& Núñez, A. 2013, AJ, 145,2

Ford, E. B., \& Rasio, F. A. 2008, ApJ, 686, 621

Freistetter, F., Krivov, A. V., \& Löhne, T. 2007, A\&A, 466, 389

Galicher, R., Rameau, J., Bonnefoy, M., et al. 2014, A\&A, 565, L4
Gauza, B., Béjar, V. J. S., Pérez-Garrido, A., et al. 2015, ApJ, 804, 96 Gizis, J. E., Allers, K. N., Liu, M. C., et al. 2015, ApJ, 799, 203

Goldreich, P., \& Ward, W. R. 1973, ApJ, 183, 1051

Golimowski, D. A., Leggett, S. K., Marley, M. S., et al. 2004, AJ, 127, 3516

Helling, C., Dehn, M., Woitke, P., \& Hauschildt, P. H. 2008, ApJ, 675, L105

Hernández, J., Calvet, N., Hartmann, L., et al. 2005, AJ, 129, 856

Hernández, J., Briceño, C., Calvet, N., et al. 2006, ApJ, 652, 472

Hewett, P. C., Warren, S. J., Leggett, S. K., \& Hodgkin, S. T. 2006, MNRAS, 367,454

Hinkley, S., Pueyo, L., Faherty, J. K., et al. 2013, ApJ, 779, 153

Horne, K. 1986, PASP, 98, 609

Ireland, M. J., Kraus, A., Martinache, F., Law, N., \& Hillenbrand, L. A. 2011, ApJ, 726, 113

Itoh, Y., Hayashi, M., Tamura, M., et al. 2005, ApJ, 620, 984

Jayawardhana, R., \& Ivanov, V. D. 2006, Science, 313, 1279

Jenkins, J. S., Pavlenko, Y. V., Ivanyuk, O., et al. 2012, MNRAS, 420, 3587

Johns-Krull, C. M., Prato, L., McLane, J. N., et al. 2016, ArXiv-eprints [arXiv: 1606.02701]

Jordi, K., Grebel, E. K., \& Ammon, K. 2006, A\&A, 460, 339

Kalas, P., Graham, J. R., Chiang, E., et al. 2008, Science, 322, 1345

Kamiaka, S., Masuda, K., Xue, Y., et al. 2015, PASJ, 67, 94

Kenyon, S. J., \& Hartmann, L. 1995, ApJS, 101, 117

Kirkpatrick, J. D., Reid, I. N., Liebert, J., et al. 2000, AJ, 120, 447

Kirkpatrick, J. D., Dahn, C. C., Monet, D. G., et al. 2001, AJ, 121, 3235

Koch, D. G., Borucki, W. J., Basri, G., et al. 2010, ApJ, 713, L79

Koen, C. 2015, MNRAS, 450, 3991

Konopacky, Q. M., Barman, T. S., Macintosh, B. A., \& Marois, C. 2013, Science, 339, 1398

Kraus, A. L., \& Ireland, M. J. 2012, ApJ, 745, 5

Kraus, A. L., Ireland, M. J., Cieza, L. A., et al. 2014, ApJ, 781, 20

Kuzuhara, M., Tamura, M., Ishii, M., et al. 2011, AJ, 141, 119

Kuzuhara, M., Tamura, M., Kudo, T., et al. 2013, ApJ, 774, 11

Lafrenière, D., Jayawardhana, R., \& van Kerkwijk, M. H. 2008, ApJ, 689, L153

Lafrenière, D., Jayawardhana, R., Janson, M., et al. 2011, ApJ, 730, 42

Lagrange, A.-M., Gratadour, D., Chauvin, G., et al. 2009, A\&A, 493, L21

Lagrange, A.-M., Bonnefoy, M., Chauvin, G., et al. 2010, Science, 329, 57

Latham, D. W., Rowe, J. F., Quinn, S. N., et al. 2011, ApJ, 732, L24

Lissauer, J. J., Jontof-Hutter, D., Rowe, J. F., et al. 2013, ApJ, 770, 131

Liu, M. C., Magnier, E. A., Deacon, N. R., et al. 2013, ApJ, 777, L20

Luhman, K. L., Adame, L., D'Alessio, P., et al. 2005a, ApJ, 635, L93

Luhman, K. L., D'Alessio, P., Calvet, N., et al. 2005b, ApJ, 620, L51

Luhman, K. L., Wilson, J. C., Brandner, W., et al. 2006, ApJ, 649, 894

Luhman, K. L., Patten, B. M., Marengo, M., et al. 2007, ApJ, 654, 570

Macintosh, B., Graham, J. R., Barman, T., et al. 2015, Science, 350, 64

Mamajek, E. E., \& Bell, C. P. M. 2014, MNRAS, 445, 2169

Mannings, V., \& Sargent, A. I. 1997, ApJ, 490, 792

Mannucci, F., Basile, F., Poggianti, B. M., et al. 2001, MNRAS, 326, 745

Marleau, G.-D., \& Cumming, A. 2014, MNRAS, 437, 1378

Marley, M. S. 2013, Science, 339, 1393

Marley, M. S., Fortney, J. J., Hubickyj, O., Bodenheimer, P., \& Lissauer, J. J. 2007, ApJ, 655, 541

Marley, M. S., Saumon, D., Cushing, M., et al. 2012, ApJ, 754, 135

Marois, C., Macintosh, B., Barman, T., et al. 2008, Science, 322, 1348

Marois, C., Zuckerman, B., Konopacky, Q. M., Macintosh, B., \& Barman, T. 2010, Nature, 468, 1080

Martín, E. L., Zapatero Osorio, M. R., Barrado y Navascués, D., Béjar, V. J. S., \& Rebolo, R. 2001, ApJ, 558, L117

Mayor, M., \& Queloz, D. 1995, Nature, 378, 355

Metchev, S. A., \& Hillenbrand, L. A. 2006, ApJ, 651, 1166

Mohanty, S., Jayawardhana, R., Huélamo, N., \& Mamajek, E. 2007, ApJ, 657, 1064

Montet, B. T., Bowler, B. P., Shkolnik, E. L., et al. 2015, ApJ, 813, L11

Moya, A., Amado, P. J., Barrado, D., et al. 2010, MNRAS, 405, L81

Muñoz, D. J., Kratter, K., Vogelsberger, M., Hernquist, L., \& Springel, V. 2015, MNRAS, 446, 2010

Mugrauer, M., \& Neuhäuser, R. 2005, Astron. Nachr., 326, 701

Mugrauer, M., Vogt, N., Neuhäuser, R., \& Schmidt, T. O. B. 2010, A\&A, 523, L1

Nagasawa, M., \& Ida, S. 2011, ApJ, 742, 72

Naud, M.-E., Artigau, É., Malo, L., et al. 2014, ApJ, 787, 5

Neuhäuser, R., \& Schmidt, T. O. B. 2012, Topics in Adaptive Optics (InTech), in press

Neuhäuser, R., Guenther, E. W., Wuchterl, G., et al. 2005, A\&A, 435, L13

Neuhäuser, R., Errmann, R., Berndt, A., et al. 2011, Astron. Nachr., 332, 547

Neuhäuser, R., Errmann, R., Raetz, S., et al. 2013, in Protostars and Planets VI Posters, 47 
Ngo, H., Knutson, H. A., Hinkley, S., et al. 2015, ApJ, 800, 138

Panić, O., Hogerheijde, M. R., Wilner, D., \& Qi, C. 2009, A\&A, 501, 269

Patience, J., King, R. R., de Rosa, R. J., \& Marois, C. 2010, A\&A, 517, A76

Peña Ramírez, K., Béjar, V. J. S., Zapatero Osorio, M. R., Petr-Gotzens, M. G., \& Martín, E. L. 2012, ApJ, 754, 30

Pecaut, M. J., Mamajek, E. E., \& Bubar, E. J. 2012, ApJ, 746, 154

Perryman, M. A. C. 2005, in Astrometry in the Age of the Next Generation of

Large Telescopes, eds. P. K. Seidelmann, \& A. K. B. Monet, ASP Conf. Ser., 338,3

Pollack, J. B., Hubickyj, O., Bodenheimer, P., et al. 1996, Icarus, 124, 62

Potter, D., Martín, E. L., Cushing, M. C., et al. 2002, ApJ, 567, L133

Preibisch, T., Brown, A. G. A., Bridges, T., Guenther, E., \& Zinnecker, H. 2002, AJ, 124, 404

Quanz, S. P., Amara, A., Meyer, M. R., et al. 2013, ApJ, 766, L1

Quanz, S. P., Amara, A., Meyer, M. R., et al. 2015, ApJ, 807, 64

Raetz, S., Schmidt, T. O. B., Czesla, S., et al. 2016, MNRAS, 460, 2834

Rameau, J., Chauvin, G., Lagrange, A.-M., et al. 2013, ApJ, 772, L15

Rasio, F. A., \& Ford, E. B. 1996, Science, 274, 954

Rayner, J. T., Cushing, M. C., \& Vacca, W. D. 2009, ApJS, 185, 289

Rebolo, R., Zapatero Osorio, M. R., Madruga, S., et al. 1998, Science, 282, 1309

Reid, I. N., \& Walkowicz, L. M. 2006, PASP, 118, 671

Reipurth, B., \& Clarke, C. 2001, AJ, 122, 432

Rieke, G. H., \& Lebofsky, M. J. 1985, ApJ, 288, 618

Safronov, V. S., \& Zvjagina, E. V. 1969, Icarus, 10, 109

Sallum, S., Follette, K. B., Eisner, J. A., et al. 2015, Nature, 527, 342

Saumon, D., \& Marley, M. S. 2008, ApJ, 689, 1327

Schmidt, T. O. B., Neuhäuser, R., Seifahrt, A., et al. 2008, A\&A, 491, 311
Schmidt, T. O. B., Neuhäuser, R., Mugrauer, M., Bedalov, A., \& Vogt, N. 2009, in 15th Cambridge Workshop on Cool Stars, Stellar Systems, and the Sun, ed. E. Stempels, AIP Conf. Ser., 1094, 852

Schmidt, T. O. B., Mugrauer, M., Neuhäuser, R., et al. 2014, A\&A, 566, A85

Schneider, J., Dedieu, C., Le Sidaner, P., Savalle, R., \& Zolotukhin, I. 2011, A\&A, 532, A79

Siess, L., Dufour, E., \& Forestini, M. 2000, A\&A, 358, 593

Skrutskie, M. F., Cutri, R. M., Stiening, R., et al. 2006, AJ, 131, 1163

Sparks, W. B., \& Ford, H. C. 2002, ApJ, 578, 543

Spiegel, D. S., \& Burrows, A. 2012, ApJ, 745, 174

Stamatellos, D., \& Whitworth, A. P. 2009, MNRAS, 392, 413

Thatte, N., Abuter, R., Tecza, M., et al. 2007, MNRAS, 378, 1229

Todorov, K., Luhman, K. L., \& McLeod, K. K. 2010, ApJ, 714, L84

Tutukov, A. V., \& Fedorova, A. V. 2012, Astron. Rep., 56, 305

van Eyken, J. C., Ciardi, D. R., von Braun, K., et al. 2012, ApJ, 755, 42

Vorobyov, E. I. 2013, A\&A, 552, A129

Wahhaj, Z., Liu, M. C., Biller, B. A., et al. 2011, ApJ, 729, 139

Weinberg, M. D., Shapiro, S. L., \& Wasserman, I. 1987, ApJ, 312, 367

Wright, J. T., Upadhyay, S., Marcy, G. W., et al. 2009, ApJ, 693, 1084

Yu, L., Winn, J. N., Gillon, M., et al. 2015, ApJ, 812, 48

Zacharias, N., Monet, D. G., Levine, S. E., et al. 2004, in BAAS 36, AAS Meeting Abstracts, 1418

Zacharias, N., Finch, C. T., Girard, T. M., et al. 2013, AJ, 145, 44

Zapatero Osorio, M. R., Béjar, V. J. S., Martín, E. L., et al. 2000, Science, 290, 103

Zuckerman, B., Rhee, J. H., Song, I., \& Bessell, M. S. 2011, ApJ, 732, 61 


\section{Appendix A: Supplementary material}
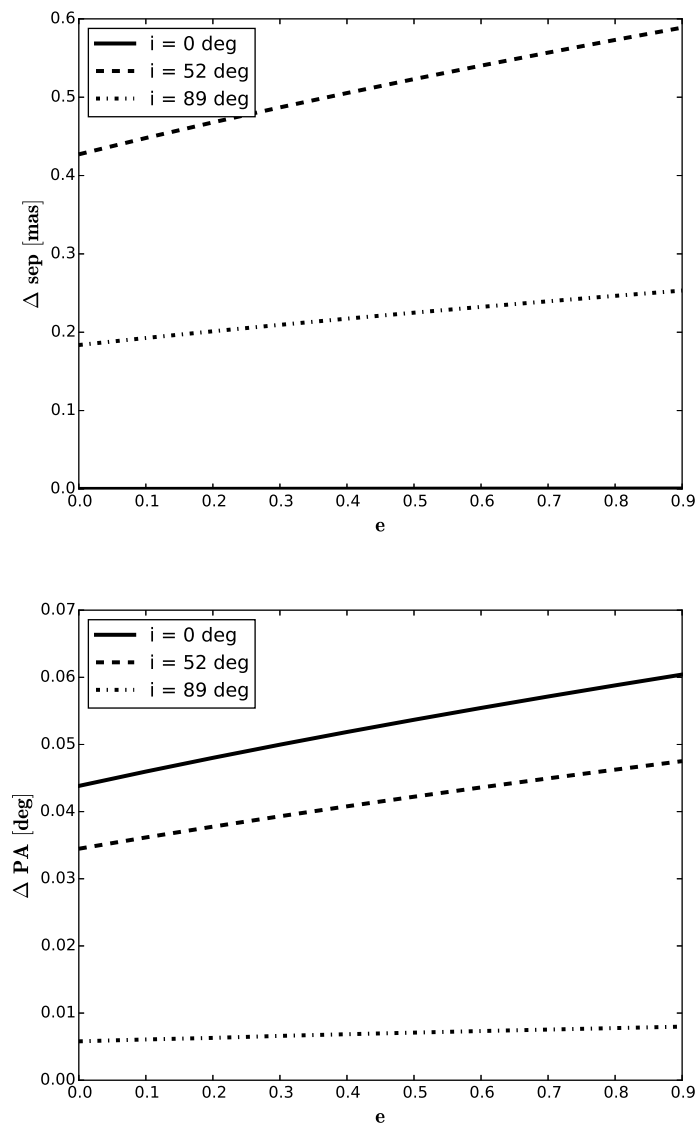

Fig. A.1. Expected maximum separation (top) and position angle (bottom) change, dependent on inclination and eccentricity of the companion for an epoch difference of three years (early in 2016, since the first calibrated epoch was made at the end of 2012).

CVSO 30 is currently not suitable for a common proper motion analysis (Table 1). Because orbital motion around the host star might be detectable, we simulated the expected maximum separation (top) and position angle (bottom) change in Fig. A.1, dependent on inclination and eccentricity of the companion for an epoch difference of three years. This corresponds to our first astrometrically calibrated epoch from 2012 to a tentative new observation early in 2016. The dedicated orbital analysis shows that even after two to three years of epoch difference, no significant orbital motion is expected for the wide companion.

A first spectrum of CVSO $30 \mathrm{c}$ at an intermediate reduction step, shown in Fig. 3 (central panel), by subtracting an average spectrum of the spike, left and right of the companions psf from the superposition of companion and spike is given in Fig. A.2. We find the results before (red spectrum) and after (blue spectrum) spike subtraction, which also removes the still-present $\mathrm{OH}$ lines. In addition, the spectrum of the host star CVSO 30 is shown in black for comparison.

In Fig. A. 3 we show the expected signal-to-noise ratio $(\mathrm{S} / \mathrm{N})$ for the given conditions and integration times (Tables 3 and 1) using ESO's exposure time calculator for SINFONI and the latest available Pickles template spectrum M6 (blue). We derive an almost identical $\mathrm{S} / \mathrm{N}$ using the flux of the companion after spike removal (Fig. 3) compared to the noise of the background next to the spike (black). However, these $\mathrm{S} / \mathrm{N}$ estimates are not

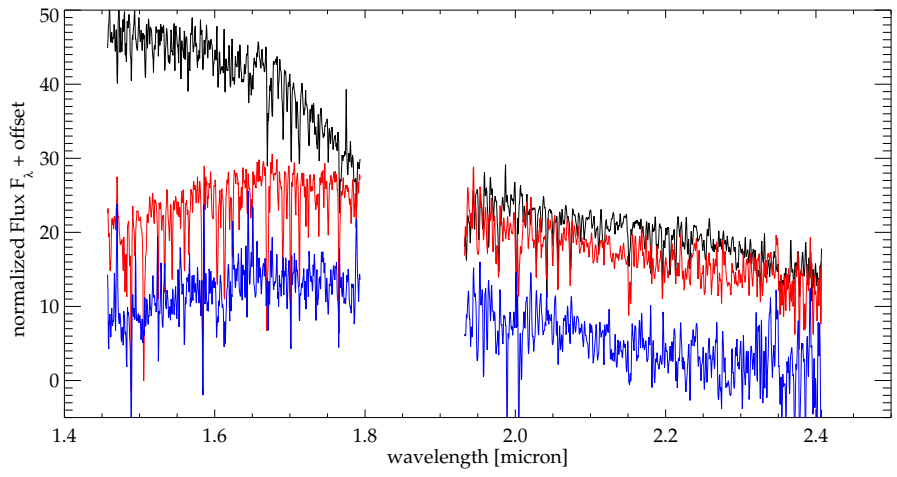

Fig. A.2. Spectrum of the primary (black) and the companion at the best illuminated pixel as given in the central panel of Fig. 3 (red; with $\mathrm{OH}$ lines), together with the spectrum after subtracting the average spike east and west of the companion (blue), which contributes about $30 \%$ of the light (beforehand). While the $H$-band spectrum presents a triangular shape and bluer colour, indicating a young sub-stellar companion, the full continuum of the companion is not reliable because different amounts of flux are superimposed by the rotating primary spike, which changes the overall continuum shape because of the different spike removal quality.

achieved for our final extracted spectrum and its noise estimate (Fig. 4) because the spike itself adds slight additional noise, and more importantly, because of the imperfect removal of the spike that dominates the final $\mathrm{S} / \mathrm{N}$ (red). To take this effect that is most likely caused by imperfect primary star positioning into account, we derived our final noise estimate, given as noise floor in Fig. 4, as the standard deviation of the neighbouring spectral channels after removing the continuum at the spectral position of interest. This noise was also used for the spectral model fitting (Figs. 4 and 5) and the reduced $\chi^{2}$ estimation for several comparison objects (Table 6).

We show the colour-magnitude diagram given in Fig. 11 in the main document with the identification of all the unlabelled objects in a full version in Fig. A.5 with the corresponding references in Table A.2. The objects seem to follow the prediction of Saumon \& Marley (2008) quite well, especially around 10 Myr. Only 2M1207 b seems to be far off, possibly because of an edgeon disk that heavily reddens the object (Mohanty et al. 2007). Whether HR $8799 \mathrm{c}$ and d are unusual can hardly be judged because no similar object with a very low luminosity is known at this age. HR 8799 b is very low in luminosity (Fig. 9), however. The younger the objects, the higher in luminosity they are at similar spectral type because of their larger radius because they are still experiencing gravitational contraction. The plot (Fig. A.5) implies that CVSO $30 \mathrm{c}$ is the first very young $(<10 \mathrm{Myr}) \mathrm{L}-\mathrm{T}$ transition object.

The core-accretion model (Safronov \& Zvjagina 1969; Goldreich \& Ward 1973; Pollack et al. 1996), was also discussed in models for HR 8799 bcde by Close (2010), who argued that the inner planet was most likely formed by core accretion, while for the outer planets the gravitational instability of the disk (Cameron 1978; Boss 1997) is the more probable formation scenario. However, HR 8799 is an A- or F-star, and recent numerical simulations (Vorobyov 2013) showed that disk fragmentation fails to produce wide-orbit companions around stars with mass $<0.7 M_{\odot}$, hence this is unfeasible for the $\sim 0.34$ $0.44 M_{\odot} \mathrm{M} 3$ star CVSO 30 . In addition, the disk would have to be large enough for in situ formation. The most massive disks around $\mathrm{M}$ stars (e.g. IM Lupi) might be large enough, but in this case, it was shown to possess almost all of its dust within $400 \mathrm{au}$ 


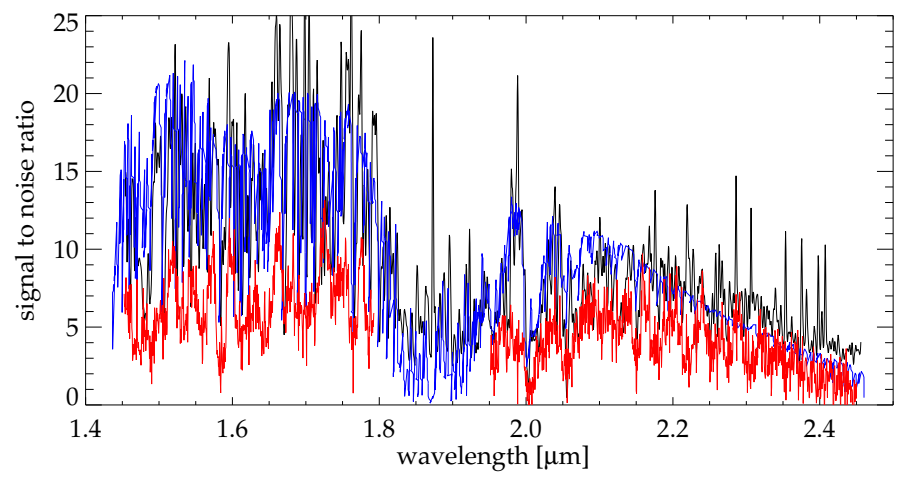

Fig. A.3. Signal-to-noise ratio $(\mathrm{S} / \mathrm{N})$ achieved for the brightest pixel vs. the background noise in the combined cube (black). For comparison the expected and almost identical $\mathrm{S} / \mathrm{N}$ is shown, simulated using the exposure time calculator (ETC) of ESO/SINFONI (blue). In red we present the final achieved $\mathrm{S} / \mathrm{N}$ of the extracted companion spectrum after removing a superimposed spike (Fig. 3), as shown in Fig. 4.

Table A.1. Evolutionary plot (Fig. 9) references.

\begin{tabular}{|c|c|c|c|}
\hline Object & Reference & Object & Reference \\
\hline GJ 504 b & Kuzuhara et al. (2013) & HD 95086 & Rameau et al. (2013) \\
\hline $2 \mathrm{M} 1207$ & Chauvin et al. (2004) & $\mathrm{b}$ & Galicher et al. (2014) \\
\hline $\mathrm{b}$ & Mohanty et al. (2007) & HR 8799 & Marois et al. (2008) \\
\hline HR 8799 & Marois et al. (2010) & $\mathrm{b}, \mathrm{c}, \mathrm{d}$ & Zuckerman et al. (2011) \\
\hline \multirow[t]{2}{*}{$\mathrm{e}$} & Zuckerman et al. (2011) & & Moya et al. (2010) \\
\hline & Moya et al. (2010) & $\beta$ Pic b & Lagrange et al. (2009) \\
\hline $1 \mathrm{RXS}$ & Lafrenière et al. (2008) & & Bonnefoy et al. (2014b) \\
\hline \multirow[t]{2}{*}{$1609 \mathrm{~b}$} & Neuhäuser \& Schmidt (2012) & & Binks \& Jeffries (2014) \\
\hline & Pecaut et al. (2012) & & Mamajek \& Bell (2014) \\
\hline CT Cha b & Schmidt et al. (2008) & CHXR 73 & Luhman et al. (2006) \\
\hline 2M044 & Todorov et al. (2010) & b & \\
\hline $144 \mathrm{~b}$ & & GQ Lup b & Neuhäuser et al. (2005) \\
\hline HD & Quanz et al. (2013) & LkCA15 & Kraus \& Ireland (2012) \\
\hline $100546 \mathrm{~b}$ & Quanz et al. (2015) & $\mathrm{b}, \mathrm{c}$ & Sallum et al. (2015) \\
\hline ROXs & Currie et al. (2014) & SR 12 & Kuzuhara et al. (2011) \\
\hline $42 \mathrm{~B} \mathrm{~b}$ & & $\mathrm{AB} \mathrm{c}$ & \\
\hline DH Tau & Itoh et al. (2005) & 2M0103 & Delorme et al. (2013) \\
\hline b & Neuhäuser \& Schmidt (2012) & $\mathrm{AB} b$ & \\
\hline \multirow[t]{2}{*}{$\mathrm{AB}$ Pic b } & Chauvin et al. (2005c) & HD & Bailey et al. (2014) \\
\hline & Neuhäuser \& Schmidt (2012) & $106906 \mathrm{~b}$ & \\
\hline \multirow[t]{2}{*}{51 Eri b } & Macintosh et al. (2015) & GU Psc b & Naud et al. (2014) \\
\hline & Montet et al. (2015) & GSC & Ireland et al. (2011) \\
\hline USco & Béjar et al. (2008) & $06214 \mathrm{~b}$ & Preibisch et al. (2002) \\
\hline CTIO & Preibisch et al. (2002) & PZ Tel B & Mugrauer et al. (2010) \\
\hline $108 \mathrm{~b}$ & Pecaut et al. (2012) & & Biller et al. (2010) \\
\hline $2 \mathrm{M} 0219 \mathrm{~b}$ & Artigau et al. (2015) & & Jenkins et al. (2012) \\
\hline
\end{tabular}

separation (Panić et al. 2009), which is still too small for formation at $662 \mathrm{au}$.

If the object has not formed in situ, a very obvious solution would be scattering induced by a stellar flyby close to the system (Adams \& Laughlin 2001; Muñoz et al. 2015) or with another object of the system. While Reipurth \& Clarke (2001) described this possibility for the formation of brown dwarfs by disintegration of a small multiple system and possibly a cutoff from the formation material reservoir, which might have occurred for example for directly imaged circumbinary planet candidates such as ROSS 458(AB) c (Burgasser et al. 2010b) or SR 12 AB c (Kuzuhara et al. 2011), the even more obvious possibility would be planet-planet scattering because an inner planet candidate CVSO $30 \mathrm{~b}$ of similar mass is present that might have been scattered inward at the very same scattering event.

A way to distinguish between the formation scenarios (Tutukov \& Fedorova 2012) would be by higher S/N ratio spectroscopy, as has been done for HR 8799 c (Konopacky et al. 2013). With both $\mathrm{H}_{2} \mathrm{O}$ and $\mathrm{CO}$ detected, it is possible to estimate the bulk atmospheric carbon-to-oxygen ratio and whether it differs from that of the primary star, which led Marley (2013)
Table A.2. Colour-magnitude plot (Figs. 11 and A.5) references.

\begin{tabular}{|c|c|c|c|}
\hline Object & Reference & Object & Reference \\
\hline 2M1207 & Chauvin et al. (2004) & HR 8799 & Marois et al. (2008) \\
\hline \multirow[t]{2}{*}{$\mathrm{A} \& \mathrm{~b}$} & Mohanty et al. (2007) & $\mathrm{b}, \mathrm{c}, \mathrm{d}$ & \\
\hline & Ducourant et al. (2008) & $\beta$ Pic b & Bonnefoy et al. (2013) \\
\hline 1RXS & Lafrenière et al. (2008) & ROXs & Kraus et al. (2014) \\
\hline $1609 \mathrm{~b}$ & & $42 \mathrm{~B} \mathrm{~b}$ & \\
\hline DH Tau & Itoh et al. (2005) & SR 12 & Kuzuhara et al. (2011) \\
\hline $\mathrm{b}$ & Luhman et al. (2006) & $\mathrm{AB} \mathrm{c}$ & \\
\hline $\begin{array}{c}\mathrm{AB} \text { Pic } \\
\mathrm{b}\end{array}$ & Chauvin et al. (2005c) & $\begin{array}{c}2 \mathrm{M} 0103 \\
\mathrm{AB} b\end{array}$ & Delorme et al. (2013) \\
\hline Ross & Burningham et al. (2011) & USco & Béjar et al. (2008) \\
\hline 458 & & CTIO & \\
\hline $\mathrm{AB} \mathrm{c}$ & & $108 \mathrm{~b}$ & \\
\hline GSC & Ireland et al. (2011) & PZ Tel b & Mugrauer et al. (2010) \\
\hline $06214 \mathrm{~b}$ & & GJ 504 & Kuzuhara et al. (2013) \\
\hline GU Psc & Naud et al. (2014) & $\mathrm{b}$ & \\
\hline $\mathrm{b}$ & & 2M0122 & Bowler et al. (2013) \\
\hline HD & Metchev \& Hillenbrand (2006) & b & \\
\hline 203030 & & HD & Potter et al. (2002) \\
\hline b & & 130948 & \\
\hline GSC & Chauvin et al. (2005b) & $\mathrm{B} \& \mathrm{C}$ & \\
\hline $08047 \mathrm{~b}$ & Bonnefoy et al. (2014a) & 2M0355 & Faherty et al. (2013) \\
\hline $\begin{array}{c}\text { HN Peg } \\
\text { b }\end{array}$ & Luhman et al. (2007) & $\begin{array}{l}\text { CD-35 } \\
2722 \text { b }\end{array}$ & Wahhaj et al. (2011) \\
\hline \multirow[t]{2}{*}{$\kappa$ And $b$} & Carson et al. (2013) & OTS 44 & Luhman et al. (2005b) \\
\hline & Hinkley et al. (2013) & Cha & Luhman et al. (2005a) \\
\hline HIP & Lafrenière et al. (2011) & 1109 & \\
\hline $78530 \mathrm{~b}$ & & HD & Bonavita et al. (2014) \\
\hline Oph 11 & Jayawardhana \& Ivanov (2006) & 284149 & \\
\hline$A \& b$ & Close et al. (2007) & $\mathrm{b}$ & \\
\hline LP & Reid \& Walkowicz (2006) & HIP & Aller et al. (2013) \\
\hline $261-75$ & Kirkpatrick et al. (2000) & $77900 \mathrm{~b}$ & \\
\hline b & & G196-3 & Rebolo et al. (1998) \\
\hline GJ 417 & Kirkpatrick et al. (2001) & $\mathrm{b}$ & \\
\hline $\mathrm{B} \& \mathrm{C}$ & Dupuy et al. (2014) & HD & Bailey et al. (2014) \\
\hline CHXR & Luhman et al. (2006) & 106906 & \\
\hline $73 \mathrm{~b}$ & & $\mathrm{~b}$ & \\
\hline $\begin{array}{c}\text { CT Cha } \\
\text { b }\end{array}$ & Schmidt et al. (2008) & $\begin{array}{l}\text { W0047 } \\
+68\end{array}$ & Gizis et al. (2015) \\
\hline $\begin{array}{l}\text { VHS } \\
1256 \mathrm{~b}\end{array}$ & Gauza et al. (2015) & $\begin{array}{c}2 \mathrm{M} 0219 \\
\mathrm{~b}\end{array}$ & Artigau et al. (2015) \\
\hline $2 \mathrm{M} 0441$ & Bowler \& Hillenbrand (2015) & PSO & Liu et al. (2013) \\
\hline $\mathrm{Bb}$ & & 318 & \\
\hline
\end{tabular}

to speculate that HR 8799 c formed by core accretion and not by gas instability.

We can place CVSO 30 c best into context by comparing it with the recent M-dwarf survey of Bowler et al. (2015), who found that fewer than $6 \%$ of $\mathrm{M}$ dwarfs harbour massive giant planets of 5-13 $M_{\text {Jup }}$ at 10-100 au and that there is currently no statistical evidence for a trend of giant planet frequency with stellar host mass at large separations. We note, however, that CVSO $30 \mathrm{c}$ would probably not have been found at the distance of their targets because it would not have been in the field of view as a result of its large separation of about 662 au. About 20 of the 49 directly imaged planet candidates at www . exoplanet . eu have an M dwarf as host star.

At a projected separation of $\sim 662$ au, the system is above the long-term stability limit of $\sim 390$ au for an M3 primary star of $0.34-0.44 M_{\odot}$ (Table 1), following the argumentation of Weinberg et al. (1987) and Close et al. (2003). However, as shown in Mugrauer \& Neuhäuser (2005), 2M1207 and its companion (Chauvin et al. 2005a) are also exceeding this long-term stability limit at about three times the age of CVSO 30.

The currently acquired data are consistent with planet-planet scattering simulations in Ford \& Rasio (2008), showing that massive planets are more likely to eject one another, whereas smaller planets are more likely to collide, resulting in stabilised systems, as supported by Kepler satellite and Doppler survey results that find predominantly smaller (Wright et al. 2009; Latham et al. 2011) low-density (e.g. Lissauer et al. 2013) planets in compact close multi-planet systems. 
T. O. B. Schmidt et al.: Direct Imaging of a second planet candidate in the transiting CVSO 30 system
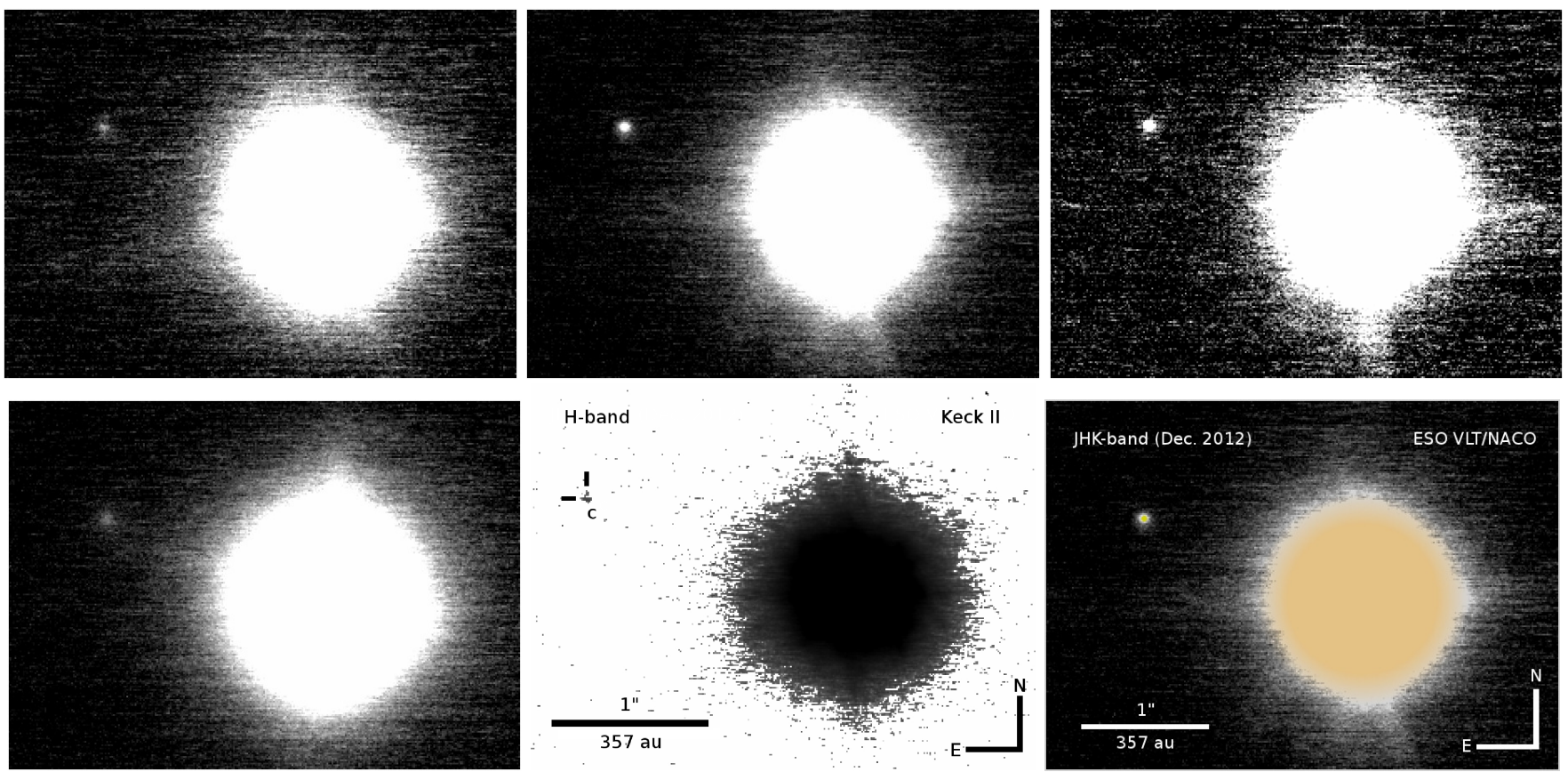

Fig. A.4. Direct images of CVSO 30 c. Top row, left to right: quasi-simultaneous VLT NACO $J$-, $H$-, and $K$ s-band data, taken in a sequence and shown in the same percentage of upper cut-off and lower cut-off value 0. Lower row, left to right: VLT NACO $J$-band with double exposure time per single image, the same in total, Keck image of data by van Eyken et al. (2012), re-reduced. We note that the companion is north-east, not a contaminant south-east, as given in van Eyken et al. (2012), and a JHKs colour composite, showing that CVSO $30 \mathrm{c}$ has similar colours as its host star (Fig. 2)

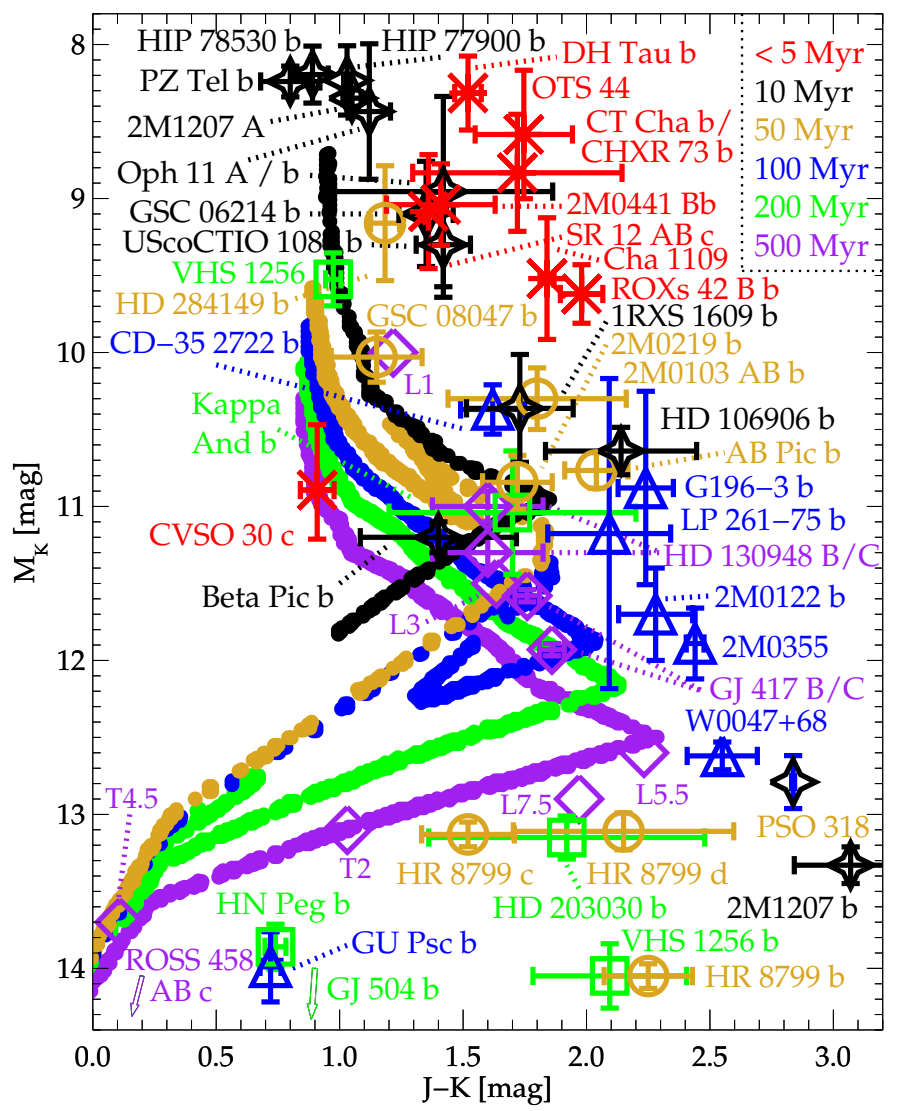

Fig. A.5. Colour-magnitude diagram of the simulated cluster brown dwarf population from Saumon \& Marley (2008). Each sequence corresponds to a different age as given in the legend. Superimposed we show the positions of several planet candidates with full identification and CVSO 30 c. See Fig. 11 and Table A.2 for further details. 